Article

\title{
Operation Optimization of Multi-District Integrated Energy System Considering Flexible Demand Response of Electric and Thermal Loads
}

\author{
Cheng Zhou ${ }^{1}$, Jianyong Zheng ${ }^{1, *}$, Sai Liu ${ }^{2}$, Yu Liu ${ }^{1}{ }^{1}$, Fei Mei ${ }^{1} \oplus$, Yi Pan ${ }^{1}$, Tian Shi ${ }^{1}$ and \\ Jianzhang $\mathbf{W u}^{\mathbf{1}}$ \\ 1 School of Electrical Engineering, Southeast University, Nanjing 210096, Jiangsu, China; \\ 220172740@seu.edu.cn (C.Z.); yuliu@seu.edu.cn (Y.L.); meifei@hhu.edu.cn (F.M.); \\ 230159517@seu.edu.cn (Y.P.); stx@seu.edu.cn (T.S.); 220182734@seu.edu.cn (J.W.) \\ 2 State Grid Jiangsu Electric Power Co., Ltd., Maintenance Branch Company, Nanjing 211102, Jiangsu, China; \\ cnsailiu@foxmail.com \\ * Correspondence: zhengjy_seu@163.com
}

Received: 13 September 2019; Accepted: 4 October 2019; Published: 10 October 2019

check for updates

\begin{abstract}
Multi-district integrated energy system (IES) can make full use of the complementary characteristics of district power and thermal system, and loads in different districts. It can improve the flexibility and economy of system operation, which has a good development prospect. Firstly, based on the general energy transfer model of the district heating network (DHN), the DHN system is described by the basic equations of the heating network and nodes considering the characteristics of the transmission time delay and heat loss in pipelines. A coupling model of DHN and multi-district IES is established. Secondly, the flexible demand response (FDR) model of electric and thermal loads is established. The load characteristics of each district in IES are studied. A shiftable load model based on the electric quantity balance is constructed. Considering the flexibility of the heat demand, a thermal load adjustment model based on the comfort constraint is constructed to make the thermal load elastic and controllable in time and space. Finally, a mixed integer linear programming (MILP) model for operation optimization of multi-district IES with the DHN considering the FDR of electric and thermal loads is established based on the supply and demand sides. The result shows that the proposed model makes full use of the complementary characteristics of electric and thermal loads in different districts. It realizes the coordinated distribution of thermal energy among different districts and improves the efficiency of thermal energy utilization through the DHN. FDR effectively reduces the peak-valley difference of loads. It further reduces the total operating cost by the coordinated operation of the DHN and multi-district IES.
\end{abstract}

Keywords: multi-district integrated energy system; complementary characteristics; district heating network; flexible demand response; operation optimization; mixed integer linear programming

\section{Introduction}

With the increasing energy demand in the industrial production and households, the contradiction has intensified between environmental problems and the energy development. Improving the energy efficiency, reducing environmental pollution, and achieving the sustainable energy development have become a major concern of academic and industrial circles in recent years [1-3]. Energy Internet [4] and integrated energy system (IES) [5] have been put forward and studied by scholars worldwide. It establishes a vision of the broad interconnection and equal sharing of energy systems in the future and provides new ideas for efficient and economic utilization of energy. At present, the planning and operation of IES is usually based on the combined cooling, heating, and power (CCHP) system in a 
single area. The equipment selection and operation management are carried out to achieve the district optimization according to load characteristics in one area [6,7]. However, the load characteristics in a specific area are often relatively monotonic, which will affect the optimization result to a certain extent [8,9]. Multi-district IES can couple the cooling and thermal loads of each area through the interconnection of the cooling and heating network. Due to the obvious interlacing phenomenon of peak and valley in each district, the complementarity of loads can be fully utilized, and the thermal output of each CCHP system can be coordinated to match the cooling and thermal demand. Finally, the optimal dispatch of energy will be realized to further improve the coordinated operation and operation economy of equipment in the system [10-12].

Establishing the interconnection model of multi-district IES and heating networks, and the operation optimization model of multi-district IES is the core of the research. In order to realize the coordinated optimization and dispatching of multi-district IES, an energy transfer model for heating networks is established, which improves the economic benefit of the IES [10]. To minimize the annual total cost and $\mathrm{CO}_{2}$ emission, a mathematical model for the selection, configuration, operation of distributed energy system, and the collaborative optimization of the district heating network (DHN) was established [13]. Fef. [14] studies the thermoelectric joint dispatching model considering the dynamic characteristics of heating pipelines and the model of heat storage devices, which promotes the economy of the system and the wind power absorption. Fef. [7] takes a commercial and residential complex in eastern Tehran as an example. A planning model is established for seven CCHP systems interconnected by the heating network, and the optimal capacity and operation strategy of the system equipment are studied. However, the heating network model is too simplified in the paper. In order to coordinate the short-term operation of power systems with large amounts of wind power and district heating systems, a combined model of transmission-constrained unit commitment with the combined electricity and DHN is established by using the linear DHN model. The heat storage capacity of the DHN is modeled by capturing the quasi-dynamics of pipeline temperature [15].

As mentioned above, the research on IES optimal operation model is carried out from the perspective of energy supply, without considering the load characteristic on its economic optimal operation. Demand response management is an important component of the future smart grid. It contributes to reducing power peak load and variation, and economic operation [16,17]. As an important zero-emission flexible resource in the power market environment, the demand response (DR) resources can effectively improve the load demand distribution and optimize the resource allocation and tap negative energy. Mining the DR resources on the load side will further improve the operation economy and flexibility of supply and demand in multi-district IES. The DR of IES can be realized by flexible electric and thermal loads. Fef. $[18,19]$ study the impact of flexible electric load on the economic benefits of the microgrid, and establishes an economic dispatching model of microgrid considering DR. An optimal operation framework for energy hub based on micro IES considering the multi-carrier load classification and scheduling, while the shiftable electrical load and curtailable thermal load scheduling model are added into the constraints of optimization formulation. It reduces the energy consumption cost, peak power, and heat load demand of the system [20]. Fef. [21] studies the two-dimensional controllability of the thermal load and establishes an economic dispatching model of the combined heat and power generation. Based on the traditional DR mechanism in recent literature, the DR optimization model of the coupled electric-thermal system is established considering the price and demand differences of various energy sources [22]. Fef. [23] suggests the combination of electric heating and intelligent energy storage such as water heaters, which can transfer energy consumption to the non-peak period and optimize the consumption price without affecting consumer's comfort. However, the above-mentioned literatures seldom take into account the complementarity of electric and thermal loads, and the load characteristic in different districts.

In order to solve the above problems, a flexible demand response (FDR) model of electric and thermal loads and an operation optimization model for multi-district IES with the DHN are established from the supply and demand sides. In the coupling part of the DHN and multi-district IES, the 
DHN system is described by the basic equations of the heating network and nodes considering the characteristics of the transmission time delay and heat loss on heating pipelines. A coupling model of DHN and multi-district IES is established. By connecting all the districts through the DHN and utilizing the complementary characteristics of loads, the surplus energy is transferred to other districts in the form of thermal power. The heat-to-electric ratios are redistributed to improve the flexibility of the system operation. In the FDR part of electric and thermal loads, the shiftable electric load model is constructed after studying the electric load characteristics in different districts of IES. Considering the flexibility of consumer's heating demand, a regulation model for thermal load based on the comfort constraint is constructed, which makes full use of the elasticity and adjustability of the thermal load in time and space. Finally, combining the FDR model with the coupling model of the DHN and multi-district IES, a mixed integer linear programming (MILP) model for operation optimization of multi-district IES considering the FDR of electric and thermal loads is established. The case result shows that the DHN realizes the complementary supply and demand of electricity and thermal loads, and promotes the coordinated distribution of thermal energy among different districts. FDR plays a role of shaving peak and filling valley. Based on the economic benefits brought by the operation of DHN and multi-district IES, the total operating cost is further reduced. The rationality and effectiveness of the proposed model are verified.

\section{Modeling of Multi-District IES}

IES is a deep integration of energy and information technology. In the process of planning, construction, control and operation, the production, transmission, transformation, distribution, storage and consumption of energy are coordinated and optimized. IES is formed as the system integrating energy production, supply and marketing of cold, heat, electricity, and natural gas. Energy Internet, energy hub [24] and ubiquitous energy Internet [25] proposed in the existing research at home and abroad are all the specific manifestations of IES. They are consistent in the basic physical architecture and equipment level. According to the description of the equipment and system architecture in IES, the basic physical architecture of IES is summarized in Figure 1.

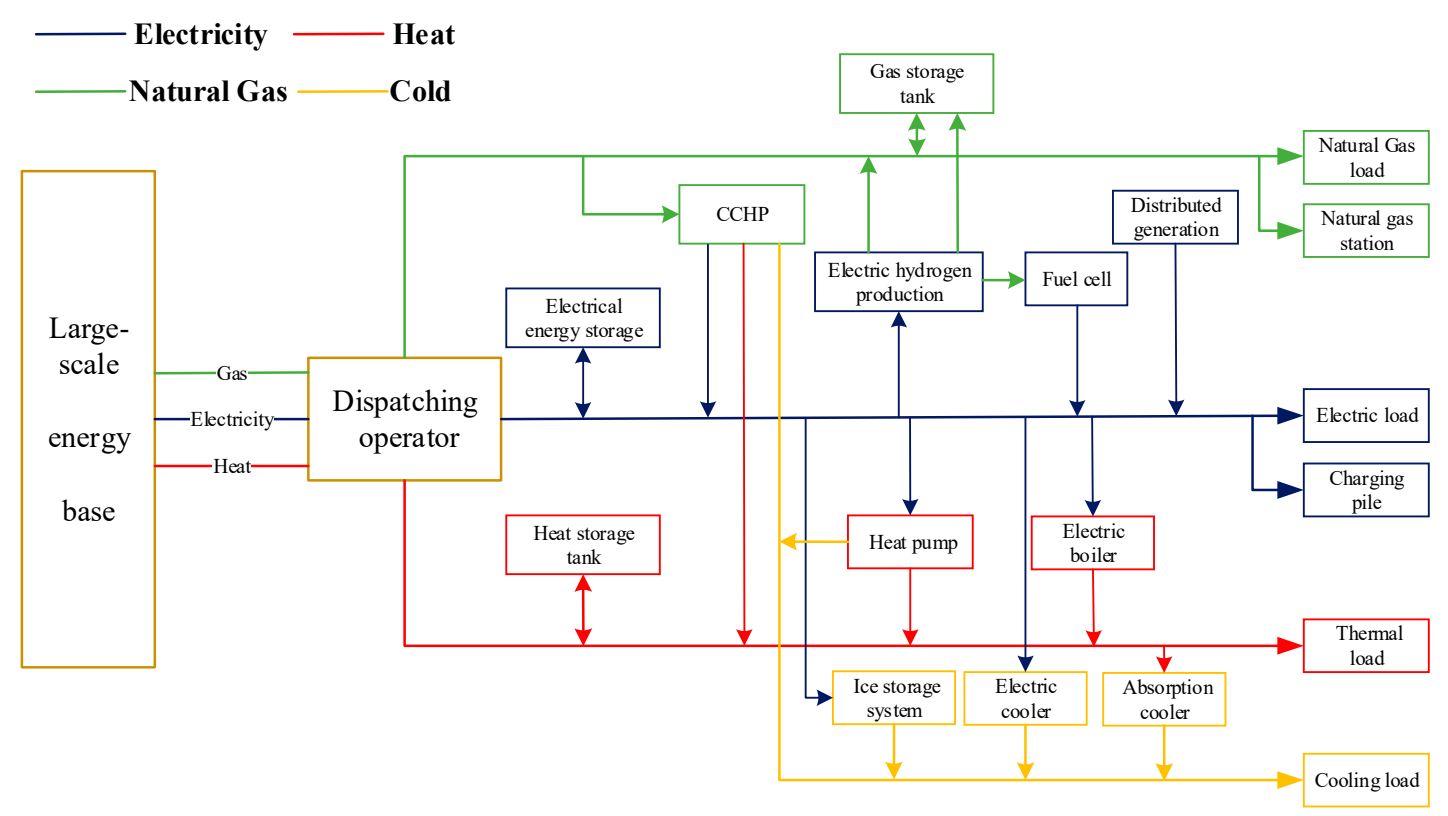

Figure 1. Structural frame of integrated energy system (IES).

According to the energy consumption/conversion of equipment, the equipment in IES can be divided into independent equipment units and coupling equipment units. Independent equipment units consume electricity, heat, gas, and cold to maintain operation. They have no coupling transformation 
and complementary utilization between heterogeneous energy flows. Coupling equipment units can realize the conversion and utilization of electricity, heat, gas, and cold.

\subsection{Model of Heating Networks}

Heating network is a system that transfers thermal energy to thermal load through pipelines and heating medium. It is essentially a fluid network which carries thermal energy. The analysis of the heating network model is mainly to study the relationship among thermal energy, temperature and heating medium. According to different heating mediums, they can be divided into hot water networks and steam networks. In China, hot water networks are mostly used. Therefore, this paper focus on the hot water heating system as an example to establish the heating network model. This paper assumes that both the water supply temperature and the return water temperature are constant, which are $90^{\circ} \mathrm{C}$ and $70{ }^{\circ} \mathrm{C}$, respectively.

The general model of heat transfer in heating networks is shown in Figure 2. It can be assumed that the connection node between CCHP $i$ and the heating network is marked as node $i$. The heating medium temperature of node $i, j$ at time $t$ is $T_{i, t}$ and $T_{j, t}$, respectively, ${ }^{\circ} \mathrm{C}$. The heating power injected to node $i$ at time $t$ is $H_{s i, t}, \mathrm{~kW}$. The flow rate of heating medium is $q_{s i, t}, \mathrm{~m}^{3} / \mathrm{s}$. The temperature is $T_{s i, t}$. The heating power of pipeline $i-j$ flowing from node $i$ to node $j$ at time $t$ is $H_{i j, t}$. The flow rate of heating medium is $q_{i j, t}$. The temperature is $T_{i j, t}$. The length of pipeline $i-j$ is $l_{i j}, \mathrm{~km}$. In the paper, the outflowing heating medium is negative and the inflowing heating medium is positive. As illustrated in Figure 2, the heating network model is mainly composed of two parts: Nodes and the network (pipelines). The node part consists of the flow balance and energy balance. The network part reflects heat loss and transmission time delay.

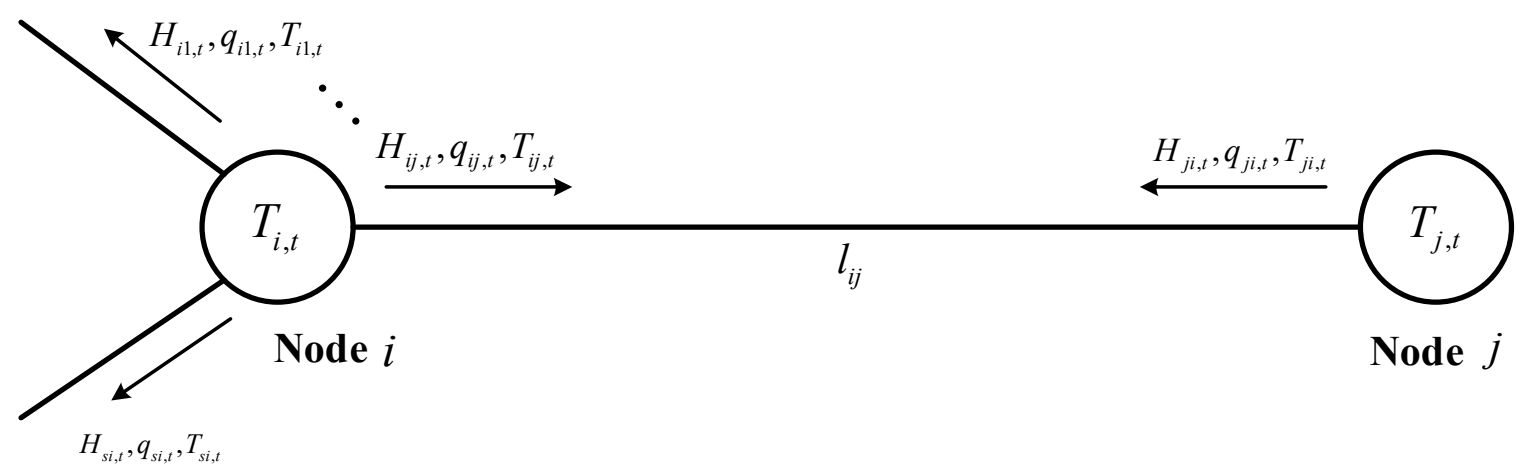

Figure 2. General energy transfer model of the heating network.

\subsubsection{Pipelines of Heating Networks}

The flow process of heating medium in pipelines is accompanied by energy transmission and loss. Based on the basic principle of heat conduction, the heat energy of heating medium in pipeline $i-j$ is:

$$
H_{i j, t}=c \rho q_{i j, t} T_{i j, t}
$$

where $H_{i j, t}, q_{i j, t}$ and $T_{i j, t}$ are the heating power, the mass flow rate and the temperature of the heating medium in pipeline $i-j$ at time $t$, respectively. $c$ is the specific heat capacity of the heating medium, $\mathrm{kJ} /\left(\mathrm{kg}^{\circ} \mathrm{C}\right) . \rho$ is the density, $\mathrm{kg} / \mathrm{m}^{3}$.

During heat transfer, the thermal characteristics of heating pipelines have a direct impact on all parts of the heating medium. The thermal characteristics of heating pipelines mainly include the transmission time delay and heat loss [26].

Transmission time delay. The heating medium absorbs heat at the heat source node to increase temperature. Heating mediums transfer to the thermal load side after increasing kinetic energy through circulating water pumps. Therefore, there is a delay effect when the heating medium flows through 
the pipeline of length $l_{i j}$. The delay time of the temperature change at both ends of pipeline $i-j$ is shown as follows:

$$
t_{\text {delay }}=\left\lceil K_{\text {delay }} \cdot \frac{l_{i j}}{v_{t}}\right\rceil,
$$

where $t_{\text {delay }}$ is the delay time of the heating medium flowing through the pipeline, s. $K_{\text {delay }}$ is the heat delay coefficient, which is related to the laying depth of pipelines. $K_{\text {delay }}$ is 1.39 in the paper [11]. $v_{t}$ is the velocity of the heating medium in the pipeline at time $t, \mathrm{~m} / \mathrm{s} .\lceil\cdot\rceil$ represents rounding up to an integer.

Heat loss [11]. The heat loss is given by the decrease of the temperature of the heating medium. The heat loss can be solved by Equation (3). Heat loss is a non-linear function related to the temperature of return water. Ignoring the influence of the return water temperature, heat loss can be approximated as follows [10]:

$$
\left\{\begin{array}{l}
H_{j i, t}=-\left(H_{i j, t-t_{\text {delay }}}-\Delta H_{i j, t_{\text {delay }}}\right) \\
\Delta H_{i j, t_{\text {delay }}}=\frac{T_{i j \text { start }, t}-T_{i j \text { out }, t}}{T_{i j \text { start }, t}-T_{i j \text { return }, t}}\left(1-\mathrm{e}^{\left.\frac{2 \pi\left(T_{i j \text { start }, t}-T_{i j \text { return }, t)}\right)}{H_{0} \sum R}\right) \approx \frac{2 \pi\left(T_{i j \text { start }, t}-T_{i j \text { out }, t}\right)}{\sum R} l_{i j}}\right.
\end{array}\right.
$$

where $H_{i j, t}, H_{i j, t-t_{\text {delay }}}$ and $\Delta H_{i j, t_{\text {delay }}}$ are thermal load in pipeline $i-j$ at time $t$, thermal power at time $t-t_{\text {delay }}$, and the heat loss in time $t_{\text {delay }} . T_{i j \text { return }, t}$ is the temperature of return water. $H_{0}$ is the thermal power in the pipeline at temperature $T_{i j s t a r t, t}$ of the heat source point. $\sum R$ is the average thermal resistance between the heating medium and the surrounding environment, $\mathrm{km}{ }^{\circ} \mathrm{C} / \mathrm{kW}$.

In addition, considering the corrosion effect and noise pollution of pipelines caused by the rapid flow rate of heating mediums, the flow rate in pipelines needs to meet the following constraints:

$$
\left|q_{i j, t}\right| \leq v_{i j, t}^{\max } S_{i j}
$$

Then,

$$
H_{i j, t}^{\max }=c \rho v_{i j, t}^{\max } S_{i j}\left(T_{i j \text { start }, t}-T_{i j \text { return }, t}\right),
$$

where $H_{i j, t}^{\max }$ and $v_{i j, t}^{\max }$ are respectively the maximum thermal power and permissible flow rate of pipeline $i-j$ at time $t$. $S_{i j}$ is the cross-sectional area of pipeline $i-j, \mathrm{~m}^{2}$.

Based on the analysis above, the model of heating networks pipelines can be described as:

$$
\left\{\begin{array}{l}
q_{i j, t}+q_{j i, t}=0 \\
H_{j i, t}=-\left(H_{i j, t-t_{\text {delay }}}-\Delta H_{i j, t_{\text {delay }}}\right) \\
\Delta H_{i j, t} \leq H_{i j, t} \leq H_{i j, t}^{\max }
\end{array}\right.
$$

The first formula ensures the continuity of heating medium flow. The remaining two formulas represent energy conservation of transmission time delay and heat loss, and the upper and lower limit constraints for thermal power of heating mediums in pipelines.

\subsubsection{Nodes of Heating Networks}

The node model is composed of the equation of the continuity of mass flow equation, and the mixture of node temperatures equation.

The equation of the continuity of mass flow is that the sum of inflow mass flow is equal to the sum of outflow mass flow at each node. Specifically, it can be characterized by the following formula:

$$
\sum_{i \in S^{\text {in }}} q_{t-t_{\text {delay }}}+\sum_{i \in S_{\text {out }}} q_{t}=0
$$


The equation of the mixture of node temperatures considers heating mediums at node $i$. Then,

$$
T_{i \text { mix }, t}=\frac{\left(c \rho \sum_{i \in S^{\text {in }}} q_{t-t_{\text {dealy }}} T_{t-t_{\text {delay }}}\right)-\sum_{i \in S^{\text {in }}} \Delta H_{t_{\text {delay }}}}{c \rho \sum_{i \in S_{\text {out }}} q_{t}},
$$

where $S^{\text {in }}$ and $S^{\text {out }}$ are pipeline sets for inflow and outflow joints of heating mediums, respectively. $q_{t-t_{\text {dealy }}}$ and $T_{t-t_{\text {delay }}}$ are the heating medium flow and temperature at time $t-t_{\text {delay }} \cdot q_{t}$ is the flow rate of outflowing heating medium. $T_{i \text { mix,t }}$ is the temperature of mixed heating mediums at node $i$.

\subsection{Coupling Structure of Heating Networks and Multi-District IES}

The structure of interconnected system for multi-district IES studied in this paper is shown in Figure 3. The system is mainly composed of CCHP system, power grid, heating network, and gas network in different IES districts. CCHP gives surplus electricity to the power grid when it is permitted by dispatching operators. The thermal power between IES in each district can be transferred to each other through the heating network. The excess heat energy in the district with abundant heat energy supply can be transferred to other districts.

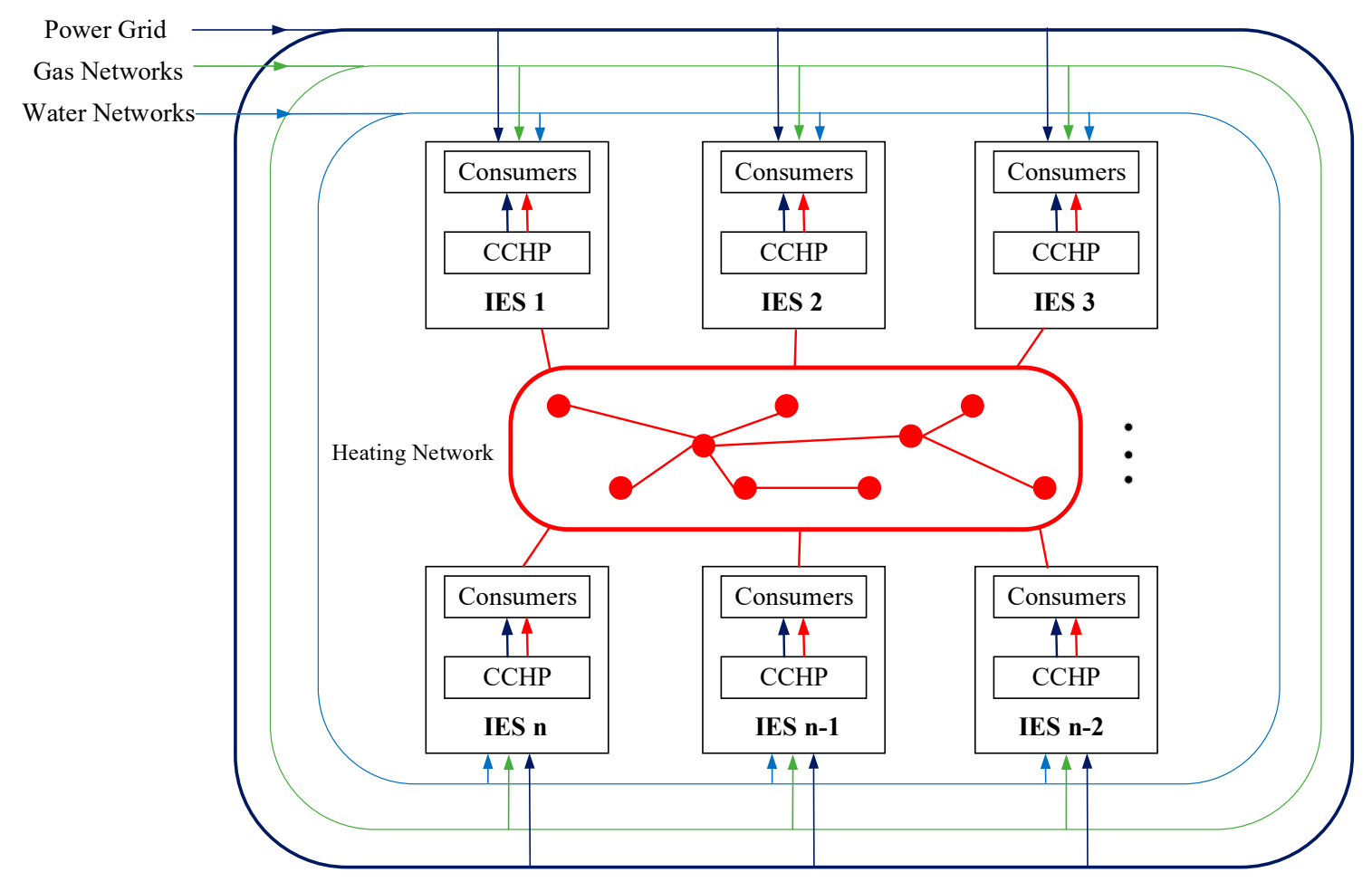

Figure 3. Coupling structure of multi-district IES and heating networks.

As shown in Figure 4, the thermal power transmission of the CCHP system is bidirectional. The bidirectional selectivity of the coupling link is realized through the automatic control of valve V1 and V2 [10]. When V1 is opened and V2 is closed, thermal power is transmitted to the heating network and connect with thermal load. When V1 is closed and V2 is opened, the heating network and CCHP system is able to connect with thermal load together. The flow of electric, thermal and cold power, and their calculation methods can be obtained from Figure 4 . 


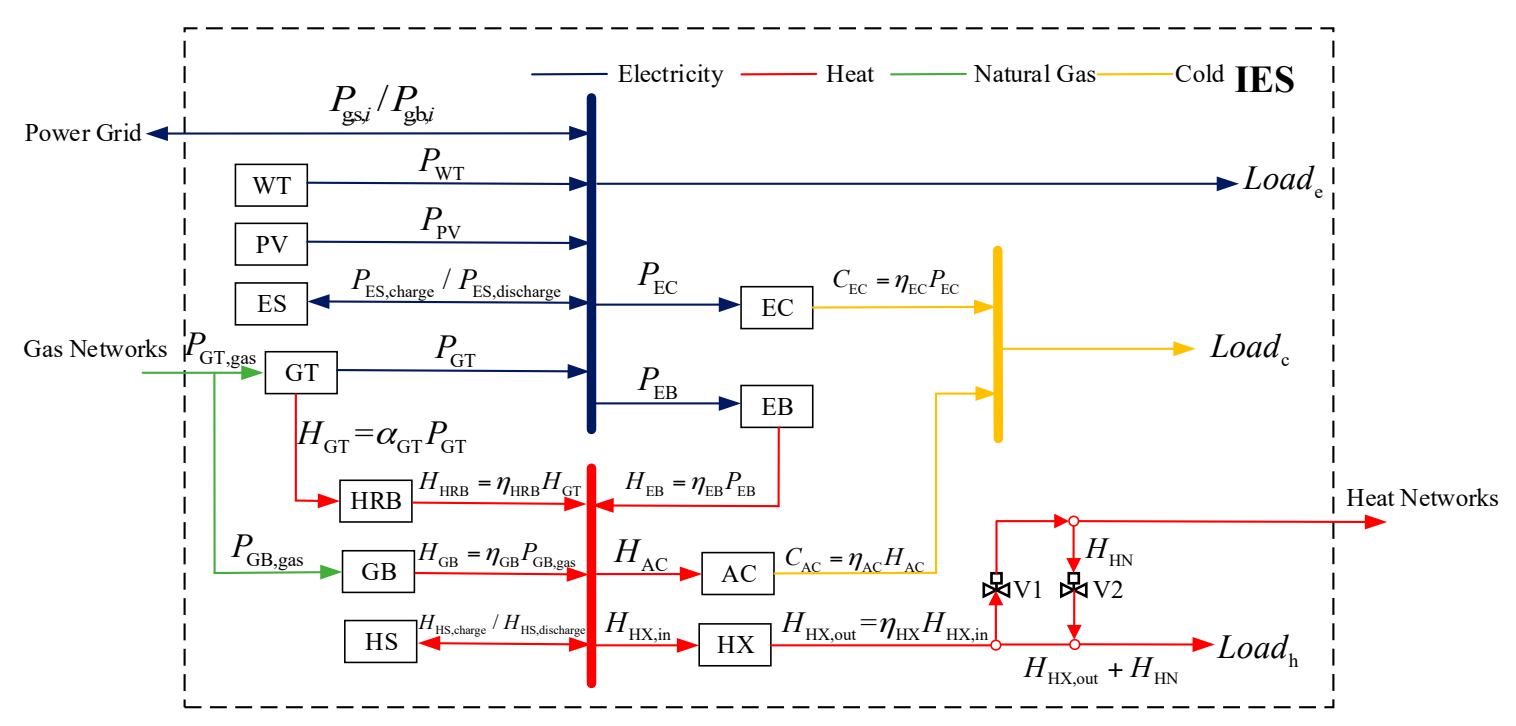

Figure 4. Coupling structure of IES and heating network.

The main equipment in IES include heat recovery boiler (HRB), heat exchange device (HX), micro gas turbine (GT), gas boiler (GB), electric boiler (EB), absorption cooler (AC), electric cooler (EC), electric energy storage (ES) and heat energy storage (HS), wind turbine (WT), photovoltaic unit (PV). The model is detailed in $[2,3]$.

\section{Flexible Demand Response of Electric and Thermal Load}

The FDR in this paper is realized by adjusting flexible electric load and flexible thermal load. Flexible electric load in IES adopts shiftable load. Thermal load includes heating load and hot water load. The FDR is based on the two-dimensional controllability of time and space. The time controllability is realized by adjusting the shiftable electric load and heating load in the operation dispatching. The space controllability is realized by the comfortable fuzziness from shiftable electric load and heating load.

\subsection{FDR Analysis of Shiftable Electric Load}

Residential consumers, industrial consumers, commercial consumers, and office consumers all have certain shiftable loads. Residential consumers can transfer load by adjusting the time of household appliances, such as using washing machines, dishwashers, water heaters in the valley period. Industrial consumers with shiftable load can be divided into three categories [27]: (a) High-energy-consuming enterprises are the main contributors of shiftable loads because of their large power consumption. Under the appropriate DR incentive, they can arrange most of their production at night. (b) Metallurgical, mechanical and textile enterprises with three shifts. The production task of the industry is continuous, so it only needs to transfer part load within a certain time without affecting the overall production process to realize peak avoidance. For example, the cement industry can carry out grinding operation in the valley period, while the raw materials of the storage bin are used in the peak period. However, the capacity of such enterprises to transfer load is limited. (c) Private and individual enterprises with flexible management can adjust their transferable loads freely because of flexible management, but their electricity consumption is relatively small.

Due to the industry nature of commercial consumers and office consumers, they are not able to transfer much load. However, they still have the potential of load transfer.

After the implementation of FDR of electric load, the load in each period is the original load plus the increasing load, and then the reduced load is subtracted, as given in Equation (9).

$$
\operatorname{Load}_{\mathrm{e}, i, t}^{\mathrm{new}}=\operatorname{Load}_{\mathrm{e}, i, t}^{\mathrm{old}}+\operatorname{Load}_{\mathrm{e}, i, t}^{\mathrm{u}}-\operatorname{Load}_{\mathrm{e}, i, t}^{\mathrm{d}}
$$


Where Load $_{e, i, t}^{\text {old }}$ and Load $d_{e, i, t}^{\text {new }}$ are respectively total load demands before and after FDR implementation. $\operatorname{Load}_{e, i, t}^{\mathrm{u}}$ and $\operatorname{Load}_{e, i, t}^{\mathrm{d}}$ are respectively load increment and load reduction of consumer $i$ at time $t$.

Usually, the total load demand depends on the household demand and production task. In the way of load transfer, consumers do not need to reduce the household demand or interrupt the production task. They can change the using time of electric energy and keep the total power consumption constant in the cycle, so the household demand and the total output are not affected. According to previous studies, there are two limitations on increasing and reducing load demand. (a) In a cycle (especially within a working day), the total power consumption remains unchanged. Load increment and reduction need to be balanced. (b) Any user has a certain uncontrollable basic load, and only part load can be adjusted. There are certain restrictions on the load increment and reduction in each time. It can be expressed as the following formula:

$$
\left\{\begin{array}{l}
\sum_{i=1}^{N} \sum_{t=1}^{M} \operatorname{Load}_{\mathrm{e}, i, t}^{\mathrm{u}}=\sum_{i=1}^{N} \sum_{t=1}^{M} \text { Load }_{\mathrm{e}, i, t}^{\mathrm{d}} \\
0 \leq \operatorname{Load}_{\mathrm{e}, i, t}^{\mathrm{u}} \leq \text { Load }_{\mathrm{e}, i, t}^{\mathrm{umax}} \\
0 \leq \text { Load }_{\mathrm{e}, i, t}^{\mathrm{d}} \leq \text { Load }_{\mathrm{e}, i, t}^{\mathrm{dmax}}
\end{array},\right.
$$

where $N$ is the number of consumers. $M$ is the total number of time periods in the scheduling cycle. $\operatorname{Load}_{e, i, t}^{\mathrm{umax}}$ and $\operatorname{Load}_{e, i, t}^{\mathrm{dmax}}$ are the maximum allowable load increment and reduction of consumer $i$ at time $t$, respectively.

\subsection{FDR Analysis of Thermal Load}

The main demand of thermal system is heating load in winter. Taking heating load model as an example, a flexible thermal load model is established.

$$
Q_{\mathrm{h}, t}=\frac{C_{\mathrm{h}} S_{\mathrm{h}}}{\Delta t}\left(T_{\mathrm{in}, t}-T_{\mathrm{in}, t-1}\right)+\mu S_{\mathrm{h}}\left(T_{\mathrm{in}, t}-T_{\mathrm{out}, t}\right),
$$

where $Q_{\mathrm{h}, t}$ is the heating load at time $t, \mathrm{~kW} . T_{\mathrm{in}, t}$ and $T_{\mathrm{out}, t}$ are respectively indoor and outdoor temperatures at time $t . C_{\mathrm{h}}$ is the heat capacity of per unit heating area, which is taken as $0.08 \mathrm{~kW} / \mathrm{m}^{2 \circ} \mathrm{C}$ in the paper. $S_{\mathrm{h}}$ is the heating area, $\mathrm{m}^{2} . \mu$ is the indoor heat loss per unit temperature difference and unit heating area, which is taken as $0.04 \mathrm{~kW} / \mathrm{m}^{2}{ }^{\circ} \mathrm{C}$.

The indoor temperature is an important index affecting heating load. The restriction of consumer's thermal comfort is the restriction of temperature range.

$$
\left\{\begin{array}{l}
T_{t}^{\min }=T_{\text {mid }, t}-\Delta T^{\max } / 2 \\
T_{t}^{\max }=T_{\operatorname{mid}, t}+\Delta T^{\max } / 2 \\
T_{\mathrm{in}, t}=T_{\mathrm{in}, t-1}+\Delta T_{\mathrm{in}, t} \\
\left|\Delta T_{\mathrm{in}, t}\right| \leq \Delta T^{\max }
\end{array},\right.
$$

where $T_{t}^{\max }$ and $T_{t}^{\min }$ are respectively the upper and lower adjusting range of indoor temperature. $T_{\mathrm{mid}, t}$ is the middle value of setting temperature range $\left[T_{t}^{\min }, T_{t}^{\max }\right] . \Delta T_{\mathrm{in}, t}$ is the changing value of indoor temperature. $\Delta T^{\max }$ is the maximum setting variable quantity of indoor temperature.

Equation (13) establishes a dynamic model of heating load, and indoor and outdoor temperature. Since the thermal load has time continuity and the indoor heating comfort demand of Equation (12) restricts the amount of temperature change at adjacent time, it is necessary to calculate the amount of thermal load change caused by the temperature difference of adjacent time. The relationship between the difference of indoor and outdoor temperature $\Delta T_{t, t-1}=T_{\mathrm{in}, t}-T_{\mathrm{in}, t-1}$ and real-time change of heat consumption $\Delta Q_{\mathrm{h}, t}$ is as follows:

$$
\Delta Q_{\mathrm{h}, t}=f\left(\Delta T_{t, t-1}\right)=f\left(T_{\mathrm{in}, t}-T_{\mathrm{in}, t-1}\right),
$$


where $\Delta T_{t, t-1}$ is the temperature difference between time $t$ and time $t-1 . \Delta Q_{\mathrm{h}, t}$ is the difference of heat consumption of time $t$ and time $t-1$.

Equations (13) and (14) can be used to calculate the indoor heat demand at different temperatures.

$$
\Delta Q_{\mathrm{h}, \mathrm{T}_{\mathrm{n}}}=f\left(\mathrm{~T}_{\mathrm{n}}\right)-f\left(\mathrm{~T}_{\mathrm{n}}-m\right),
$$

where $\Delta Q_{\mathrm{h}, \mathrm{Tn}}$ is the heat required to raise indoor temperature by $m^{\circ} \mathrm{C}$.

Load characteristics of the thermal system has the following load characteristics. Firstly, temperature is its regulation scale with a low consumer's sensitivity, so temperature only needs to be assured in the comfort zone. Secondly, affected by the regulation means and network characteristics, thermal system has a slow regulation rate relative to power system dispatching. The building envelope and network structure have a certain heat storage capacity, which makes it strong enough to suppress and tolerate short-term fluctuations and intermittences. Flexible thermal load regulation is as follows:

$$
P_{\mathrm{h}, t}^{\mathrm{FDR}}=\alpha_{\mathrm{h}, t}^{\mathrm{FDR}} H_{t}^{\mathrm{FDR}, \max }, \alpha_{\mathrm{h}, t}^{\mathrm{FDR}} \in[0,1],
$$

where $P_{\mathrm{h}, t}^{\mathrm{FDR}}$ is the controlled quantity of thermal load. $\alpha_{\mathrm{h}, t}^{\mathrm{FDR}}$ is the regulation rate. $\alpha_{\mathrm{h}, t}^{\mathrm{FDR}}>0$ indicates that the regulation is in progress and $\alpha_{\mathrm{h}, t}^{\mathrm{FDR}}=0$ indicates that the regulation is interrupted. $H_{t}^{\mathrm{FDR}, \max }$ represents the maximum FDR controlled quantity of thermal load at time $t$. FDR dispatch of thermal system should satisfy the constraints of consumer's comfort, which needs to constrain the regulating range of indoor temperature. The maximum constraints of FDR regulation can be calculated by Equations (14) and (15).

$$
P_{\mathrm{h}, t}^{\mathrm{FDR}} \leq \Delta Q_{t}^{\mathrm{FDR}, \max },
$$

where $\Delta Q_{t}^{\mathrm{FDR}, \max }$ is the maximum controlled quantity under temperature constraints, which can be calculated by Equations (13)-(15).

The DR of thermal load is to increase the controlled quantity as much as possible and promote the flexible adjustment ability of thermal load under the condition of satisfying the consumer's satisfaction and the controlled restriction of thermal load. In this paper, the calculation method of adjustment cost for thermal load is given by:

$$
C_{\mathrm{h}}^{\mathrm{FDR}}=\sum_{i=1}^{N} \sum_{t=1}^{M} b_{\mathrm{h}}^{\mathrm{FDR}} P_{\mathrm{h}, i, t}^{\mathrm{FDR}},
$$

where $C_{\mathrm{h}}^{\mathrm{FDR}}$ is the compensation cost for thermal load FDR. $b_{\mathrm{h}}^{\mathrm{FDR}}$ is the compensation unit price. $P_{\mathrm{h}, i, t}^{\mathrm{FDR}}$ is the regulation amount of thermal load of IES $i$ at time $t$. Based on Weber-Fechner law, this paper constructs a model of consumers' willingness to participate in FDR of thermal load. Through the empirical calculation of this law, a more reasonable and effective pricing scheme is proposed to meet the psychological expectation. Therefore, the relationship between the real-time compensation price of FDR and real-time electricity price is as follows:

$$
b_{\mathrm{h}}^{\mathrm{FDR}}=b_{\mathrm{h}} \ln \left(c_{\mathrm{h}}\right)+C,
$$

where $b_{\mathrm{h}}$ is the compensation coefficient of thermal load FDR, whose value is $0.5 . c_{\mathrm{h}}$ is the selling price of thermal load, the value is 0.8 .

\section{Operation Optimization Model of Multi-District IES Considering FDR of Electric and Thermal Load}

The optimization of the coupled system of heating network and multi-district IES is based on the power constraints, operation constraints of heating networks, and main equipment. Considering the FDR control of electric and thermal power, an economic optimal dispatching model for multi-district IES is proposed. 


\subsection{Objective Function}

The optimization objective function includes the cost of purchasing electricity from the power grid, the profit of selling electricity to the power grid, the natural gas cost, the operation cost of the heating network and the compensation cost of FDR for thermal load. The formula is as follows:

$$
\min C_{\mathrm{MD}-\mathrm{IES}}=\mathrm{C}_{\mathrm{e}, \mathrm{b}}-\mathrm{C}_{\mathrm{e}, \mathrm{s}}+C_{\mathrm{gas}}+C_{\mathrm{HN}}+C_{\mathrm{h}}^{\mathrm{FDR}},
$$

where $C_{\mathrm{e}, \mathrm{b}}, C_{\mathrm{e}, \mathrm{s}}, C_{\mathrm{gas}} C_{\mathrm{HN}}$ and $C_{\mathrm{h}}^{\mathrm{FDR}}$ are the cost of purchasing electricity from the power grid, the profit of selling electricity to the power grid, the natural gas cost, the operation cost of the heating network, and the compensation cost of FDR for thermal load, respectively, $¥$.

$$
C_{\mathrm{e}, \mathrm{b}}=\sum_{i=1}^{N} \sum_{t=1}^{M}\left(c_{\mathrm{e}, \mathrm{b}, t} P_{\mathrm{g}, \mathrm{b}, i, t} \Delta t\right)
$$

where $N$ is the number of districts in multi-district IES. $M$ is the total number of scheduling periods. $c_{\mathrm{e}, \mathrm{b}, t}$ is the purchase price of electricity at time $t, ¥ /(\mathrm{kWh}) . P_{\mathrm{g}, \mathrm{b}, i, t}$ is the power purchased from the grid of district $i$ at time $t, \mathrm{~kW}$. $\Delta t$ is time of each scheduling period, $\mathrm{h}$.

$$
C_{\mathrm{e}, \mathrm{s}}=\sum_{i=1}^{N} \sum_{t=1}^{M}\left(c_{\mathrm{e}, \mathrm{s}, t} P_{\mathrm{g}, \mathrm{s}, i, t} \Delta t\right)
$$

where $c_{\mathrm{e}, \mathrm{s}, \mathrm{t}}$ is the unit price of selling electricity, $¥ /(\mathrm{kWh}) . P_{\mathrm{g}, \mathrm{b}, i, t}$ is the power sold to the grid, $\mathrm{kW}$.

The natural gas cost is as follows:

$$
C_{\text {gas }}=c_{\text {gas }} \sum_{i=1}^{N} \sum_{t=1}^{M}\left(P_{\mathrm{GT}, \text { gas }, i, t}+P_{\mathrm{GB}, \text { gas }, i, t}\right) \Delta t=c_{\text {gas }} \sum_{i=1}^{N} \sum_{t=1}^{M}\left(\frac{P_{\mathrm{GT}, i, t}}{\eta_{\mathrm{GT}, i}}+\frac{Q_{\mathrm{GB}, i, t}}{\eta_{\mathrm{GB}, \mathrm{i}}}\right) \Delta t,
$$

where $c_{\text {gas }}$ is the unit calorific value price of natural gas, $¥ /(\mathrm{kWh}) . P_{\mathrm{GT}, g a s, i, t}$ and $P_{\mathrm{GB}, \text { gas }, i, t}$ are respectively the electric and thermal power of GT and GB in district $i$ at time $t, \mathrm{~kW} . \eta_{G T, i}$ and $\eta_{G B, i}$ are respectively the efficiencies of GT and GB in district $i$.

The operation cost of the heating network is mainly the electricity cost of circulating pumps in pipelines, which can be estimated by ratios of electricity consumption to transfer heat quantity [28].

$$
C_{\mathrm{HN}}=\sum_{i=1}^{R} \sum_{t=1}^{M}\left(E H R_{i} c_{\mathrm{e}, \mathrm{b}, t} H_{\mathrm{HN}, i, t} \Delta t\right),
$$

where $R$ is the number of circulating pumps. $E H R_{i}$ is the ratio of electricity consumption to transfer heat quantity of pump $i$. $H_{\mathrm{HN}, i, t}$ is the heat distributed by pump $i, \mathrm{~kW}$.

The formula for calculating the compensation cost of FDR of thermal load is shown in Equation (17).

\subsection{Operation Constraints}

For the IES structure shown in Figure 4, the constraints mainly include the energy balance constraints of the electric, thermal, and cooling bus, the output constraints of CCHP system in multi-district IES, the FDR constraints, the heating network constraints and the coupling constraints of the heating network and multi-district IES.

Considering the affiliated status of energy conversion devices and the diversity of energy conversion forms, relevant literature usually merge energy conversion devices into source equipment with energy efficiency when modeling CCHP system [29]. In this paper, the bus structure is adopted, which can independently model different power sources, thermal sources, energy storages, conversion 
devices for electric, thermal, and cooling loads. At the same time, it is easy to write system equilibrium equations and equipment constraints.

\subsubsection{Energy Balance Constraints of Bus}

The balance equation of electric bus is as follows:

$$
P_{\mathrm{g}, \mathrm{b}, i, t}-P_{\mathrm{g}, \mathrm{s}, i, t}+P_{\mathrm{WT}, i, t}+P_{\mathrm{PV}, i, t}+P_{\mathrm{ES}, \text { discharge }, i, t}-P_{\mathrm{ES}, \text { charge }, i, t}+P_{\mathrm{GT}, i, t}=\operatorname{Load}_{\mathrm{e}, i, t}^{\text {new }}+P_{\mathrm{EC}, i, t}+P_{\mathrm{EB}, i, t}
$$

where $P_{\mathrm{g}, \mathrm{b}, i, t}$ and $P_{\mathrm{g}, \mathrm{s}, i, t}$ are respectively the purchasing and selling electric power with the superior power grid of district $i$ at time $t . P_{\mathrm{WT}, i, t}, P_{\mathrm{PV}, i, t}, P_{\mathrm{ES}, \text { discharge }, i, t}, P_{\mathrm{ES}, \text { charge } i, t, t}$ and $P_{\mathrm{GT}, i, t}$ are the outputs of $\mathrm{WT}$ and $\mathrm{PV}$, the charging and discharging power of $\mathrm{ES}$, and the output of GT of district $i$ at time $t$. Load $_{e, i, t}^{\text {new }}, P_{\mathrm{EC}, i, t}$ and $P_{\mathrm{EB}, i, t}$ are the electric load, the power of EC and $\mathrm{EB}$, respectively.

The balance equation of thermal bus is as follows:

$$
\eta_{\mathrm{HRB}, i} \alpha_{\mathrm{GT}, i} P_{\mathrm{GT}, i, t}+\eta_{\mathrm{GB}, i} P_{\mathrm{GB}, \mathrm{gas}, i, t}+\eta_{\mathrm{EB}, i} P_{\mathrm{EB}, i, t}+H_{\mathrm{HS}, \text { discharge }, i, t}-H_{\mathrm{HS}, \text { charge }, i, t}=H_{\mathrm{AC}, i, t}+H_{\mathrm{HX}, \text { in }, i, t}
$$

where $\eta_{\mathrm{HRB}, i}, \eta_{\mathrm{GB}, i}, \eta_{\mathrm{EB}, i}$ and $\alpha_{\mathrm{GT}, i}$ are the transformation efficiencies of $\mathrm{HRB}, \mathrm{GB}$, and $\mathrm{EB}$, and the heat-to-electric ratio of GT in district $i$, respectively. $P_{\mathrm{GBgas}, i, t}, H_{\mathrm{HS} \text {, discharge }, i, t}, H_{\mathrm{HS}}$, charge $i, i, H_{\mathrm{AC}, i, t}$ and $H_{\mathrm{HX}, \text { in }, i, t}$ are natural gas consumed by GB, releasing and storing thermal power of $\mathrm{HS}$, thermal power consumed by AC and HX in district $i$ at time $t$, respectively.

The balance equation of cooling bus is as follows:

$$
\eta_{\mathrm{EC}, i} P_{\mathrm{EC}, i, t}+\eta_{\mathrm{AC}, i} H_{\mathrm{AC}, i, t}=\operatorname{Load}_{\mathrm{c}, i, t}
$$

where $\eta_{\mathrm{EC}, i}$ and $\eta_{\mathrm{AC}, i}$ are the transformation efficiencies of EC and AC. Load $_{\mathrm{c}, i, t}$ is the cooling load in district $i$ at time $t$.

\subsubsection{Output Constraints of CCHP System in Multi-District IES}

In addition to the bus energy balance equation of the system, the constraints of various types of equipment including the upper and lower output limits, charging and discharging power and energy constraints of energy storage equipment should also be considered.

The electrical, thermal, and cooling power of each equipment meets the requirements of upper and lower limits:

$$
\left\{\begin{array}{l}
P_{i}^{\min } \leq P_{i} \leq P_{i}^{\max } \\
H_{i}^{\min } \leq H_{i} \leq H_{i}^{\max } \\
C_{i}^{\min } \leq C_{i} \leq C_{i}^{\max }
\end{array},\right.
$$

where $P_{i}, H_{i}$ and $C_{i}$ are electrical, thermal, and cooling power of equipment $i$, respectively. $P_{i}^{\min }, P_{i}^{\max }$, $H_{i}^{\min }, H_{i}^{\max }, C_{i}^{\min }$ and $C_{i}^{\max }$ are the lower and upper limits of electrical, thermal, and cooling power of equipment $i$, respectively.

For energy storage equipment, the operation constraints include charging and discharging power, storage energy and other constraints.

$$
\left\{\begin{array}{l}
0 \leq P_{\mathrm{ES}, \text { charge }, i, t} \leq \gamma_{\mathrm{ES}, \text { charge }, i}^{\max } \operatorname{Cap}_{\mathrm{ES}, i} \\
0 \leq P_{\mathrm{ES}, \text { discharge }, i, t} \leq \gamma_{\mathrm{ES}, \text { discharge }, i}^{\operatorname{ma}} \operatorname{Cap}_{\mathrm{ES}, i} \\
P_{\mathrm{ES}, \text { charge }, i, t} P_{\mathrm{ES}, \text { discharge }, i, t}=0 \\
E_{\mathrm{ES}, i, t}=\left(1-\sigma_{\mathrm{ES}, i}\right) E_{\mathrm{ES}, i, t-1}+\left(\eta_{\mathrm{ES}, \text { charge }, i} P_{\mathrm{ES}, \text { charge }, i, t}+\frac{P_{\mathrm{ES}, \text { discharge }, i t}}{\eta_{\mathrm{ES}, \text { discharge }, i}}\right) \Delta t \\
E_{\mathrm{ES}, i}^{\min } \leq E_{\mathrm{ES}, i, t} \leq E_{\mathrm{ES}, i}^{\max } \\
E_{\mathrm{ES}, i, 0}=E_{\mathrm{ES}, i, \mathrm{~T}}
\end{array}\right.
$$


where $\gamma_{\mathrm{ES}, \text { charge }, i}^{\operatorname{and}} \gamma_{\mathrm{ES} \text {,discharge, } i}^{\max }$ are respectively the maximum ratios of charging and discharging of ES $i$. The third formula guarantees charging and discharging are not at the same time. $E_{\mathrm{ES}, i, t}$ is the stored energy of ES $i$ at time $t, \mathrm{kWh}$. Cap $\mathrm{ES}, i_{i}$ is the maximum capacity of $\mathrm{ES} i, \mathrm{kWh} . \sigma_{\mathrm{ES}, \mathrm{i}}, \eta_{\mathrm{ES}, \text { charge }, i}$ and $\eta_{\mathrm{ES}, \text { discharge, } i}$ are respectively the self-discharge rate, and efficiencies of charging and discharging of ES $i$. $E_{\mathrm{ES}, i}^{\max }$ and $E_{\mathrm{ES}, i}^{\min }$ are respectively the maximum and minimum stored energy of ES $i$. The fifth formula indicates that the stored energy remains unchanged before and after the scheduling cycle.

The FDR constraints are detailed in Equations (9)-(16). The constraints of the heating network are detailed in Equation (6).

\subsubsection{Coupling Constraints of the Heating Network and Multi-District IES}

Compared with IES without heating networks, multi-district IES with DHN adds the energy coupling link between CCHP system and the heating network at the output port of HX. The corresponding coupling constraint is the energy coupling equation as follows:

$$
H_{\mathrm{HX}, \mathrm{out}, i, t}+H_{\mathrm{HN}, i, t}=\operatorname{Load}_{\mathrm{h}, i, t}
$$

where, $H_{\mathrm{HX}, \mathrm{out}, i, t} H_{\mathrm{HN}, i, t}$ and $\operatorname{Load}_{\mathrm{h}, i, t}$ are the thermal power of $\mathrm{HX}$, thermal power obtained from the heating network and thermal load of the system.

The optimization model consists of three parts: The optimization of energy flow in each IES district, FDR control for electric and thermal load, and energy flow paths in the heating network. They are coupled through thermal power between multi-district IES and the heating network.

\subsection{Model Solution}

In this paper, a 0-1 MILP method is used to solve the optimization model. The standard form of the model is as follows:

$$
\left\{\begin{array}{lll}
\min & \boldsymbol{c}^{\mathrm{T}} \boldsymbol{x} & \\
\text { s.t. } & \boldsymbol{A} \boldsymbol{x}=\boldsymbol{b} \\
& x_{\min } \leq x_{i} \leq x_{\max }, \quad i \in \boldsymbol{I} \\
& x_{j} \in\{0,1\}, \quad j \in \boldsymbol{J}
\end{array},\right.
$$

The decision variables include the output of CCHP units in each district, the real-time storing and releasing power of electric and thermal energy storage devices, the purchasing and selling electricity with the power grid, the output of conversion equipment and the thermal power flow in the pipelines of the heating network. Equality constraints and inequality constraints are shown in Table 1. Since the constraints include coupling variables, such as the storing and releasing power of electric and thermal energy storage devices, and the purchasing and selling electricity, the 01 variable is introduced into the model.

Table 1. Details of constraints.

\begin{tabular}{ccccc}
\hline Constraints Type & IES & FDR & Heating Networks & Coupling Relation \\
\hline $\begin{array}{c}\text { Equality } \\
\text { constraints }\end{array}$ & $\begin{array}{c}\text { (1) Energy balance equation of bus } \\
\text { (2) Energy relationship of energy } \\
\text { storage devices }\end{array}$ & $\begin{array}{c}\text { (1) Equation } \\
\text { constraints of } \\
\text { electric load } \\
\text { of CCHP system }\end{array}$ & $\begin{array}{c}\text { (1) Equilibrium equation of node } \\
\text { (2) Heat loss equation of } \\
\text { pipelines }\end{array}$ & $\begin{array}{c}\text { (1) Energy coupling } \\
\text { equation of the } \\
\text { heating network } \\
\text { and CCHP system }\end{array}$ \\
\hline $\begin{array}{c}\text { Inequality } \\
\text { constraints }\end{array}$ & $\begin{array}{c}\text { (1) Operating constraints of CCHP } \\
\text { system }\end{array}$ & $\begin{array}{c}\text { (1) Flexible control } \\
\text { constraints of } \\
\text { thermal load }\end{array}$ & $\begin{array}{c}\text { (1) Input and output power } \\
\text { constraints of pipelines }\end{array}$ \\
\hline
\end{tabular}

At present, there are mature algorithms to solve the model, which can be directly solved by commercial software such as Cplex, Gurobi, Lingo, etc. Based on Yalmip and Cplex tool in MATLAB 2015a, a 0-1 MILP program is developed to solve the problem in the paper. 


\section{Case Analysis}

\subsection{Case Description}

In this paper, the coupled system of multi-district IES with DHN is taken as a case as shown in Figure 5. The IES is divided into four sub-districts: Residential district, commercial district, office district, and industrial district. The CCHP system is built in each district. The CCHP system structure for each sub-district is shown in Figure 4. Electric and thermal energy storage devices are equipped in each district. The capacity of each equipment is shown in Table 2. The parameters of the heating network are given in Table 3. The running cost of the heating network in the simulation is calculated according to the thermal power distributed by pipelines. Detailed equipment parameters in each sub-districts are given in Table 4.

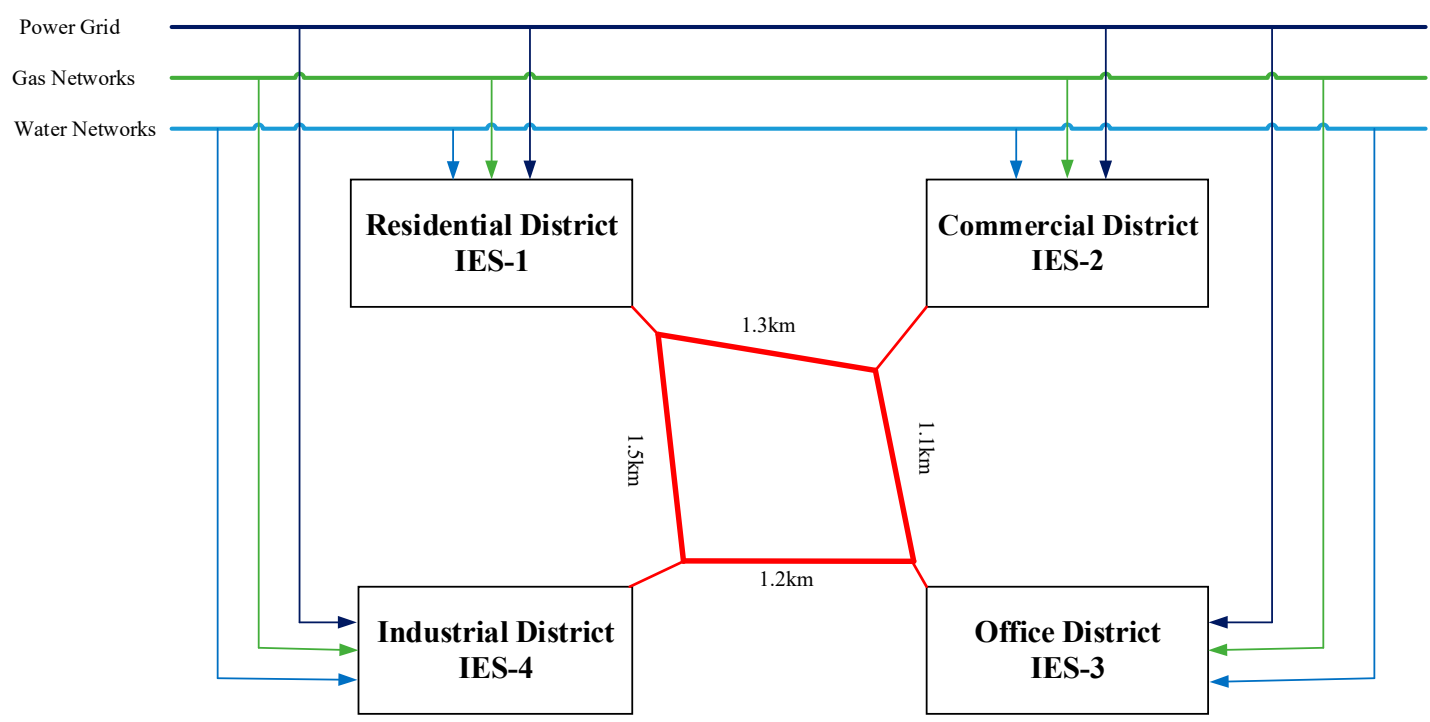

Figure 5. Coupling structure between the multi-district IES and the heating network.

Table 2. Capacities of devices in each sub-district.

\begin{tabular}{ccccc}
\hline \multirow{2}{*}{ Devices } & \multicolumn{4}{c}{ Capacities of Devices/(kW) } \\
\cline { 2 - 5 } & IES-1 & IES-2 & IES-3 & IES-4 \\
\hline WT & 1000 & 2500 & 1600 & 5000 \\
PV & 500 & 1200 & 1000 & 2000 \\
GT & 2000 & 8000 & 3000 & 15,000 \\
HRB & 5000 & 15,000 & 8000 & 30,000 \\
GB & 5000 & 5000 & 5000 & 10,000 \\
EB & 5000 & 5000 & 5000 & 10,000 \\
HX & 10,000 & 20,000 & 10,000 & 40,000 \\
EC & 5000 & 5000 & 5000 & 10,000 \\
AC & 5000 & 5000 & 5000 & 10,000 \\
\hline \multirow{2}{*}{ Devices } & \multicolumn{5}{c}{ Capacities of Devices/(kWh) } \\
\cline { 2 - 5 } & IES-1 & IES-2 & IES-3 & IES-4 \\
\hline ES & 1000 & 1000 & 1000 & 2000 \\
HS & 2000 & 2000 & 2000 & 6000 \\
\hline
\end{tabular}


Table 3. Parameters of the heating network.

\begin{tabular}{cccccc}
\hline Pipeline & Length/(km) & Diameter/(m) & $\begin{array}{c}\text { Maximum Flow } \\
\text { Velocity/(m/s) }\end{array}$ & $\left.\sum \mathbf{R} / \mathbf{( k m} \cdot{ }^{\circ} \mathbf{C} / \mathbf{k W}\right)$ & EHR \\
\hline $1-2$ & 1.3 & 0.3 & 3 & 20 & 0.0063 \\
$2-3$ & 1.1 & 0.3 & 3 & 20 & 0.0059 \\
$3-4$ & 1.2 & 0.3 & 3 & 20 & 0.0069 \\
$4-1$ & 1.5 & 0.3 & 3 & 20 & 0.0058 \\
\hline
\end{tabular}

Table 4. Equipment parameters of IES in each sub-district.

\begin{tabular}{ccccc}
\hline Parameters & IES-1 & IES-2 & IES-3 & IES-4 \\
\hline$P_{\mathrm{GT}}^{\min } /(\mathrm{kW})$ & 120 or OFF & 450 or OFF & 200 or OFF & 800 or OFF \\
$\alpha_{\mathrm{GT}}$ & 2.3 & 2.3 & 2.3 & 2.3 \\
$\eta_{\mathrm{GT}}$ & 0.3 & 0.3 & 0.3 & 0.3 \\
$\eta_{\mathrm{HRB}}$ & 0.73 & 0.73 & 0.73 & 0.73 \\
$\eta_{\mathrm{GB}}$ & 0.9 & 0.9 & 0.9 & 0.9 \\
$\eta_{\mathrm{EB}}$ & 0.9 & 0.9 & 0.9 & 0.9 \\
$\eta_{\mathrm{EC}}$ & 4 & 4 & 4 & 4 \\
$\eta_{\mathrm{AC}}$ & 1.2 & 1.2 & 1.2 & 1.2 \\
$\eta_{\mathrm{HX}}$ & 0.9 & 0.9 & 0.9 & 0.9 \\
$\gamma_{\mathrm{ES}, \text { charge }}^{\max }$ & 0.2 & 0.2 & 0.2 & 0.2 \\
$\gamma_{\mathrm{ES} \text {,discharge }}^{\max }$ & 0.4 & 0.4 & 0.4 & 0.4 \\
$\eta_{\mathrm{ES}, \text { charge }}$ & 0.95 & 0.95 & 0.95 & 0.95 \\
$\eta_{\mathrm{ES}, \text { discharge }}$ & 0.95 & 0.95 & 0.95 & 0.95 \\
$\sigma_{\mathrm{ES}}$ & 0.04 & 0.04 & 0.04 & 0.04 \\
$E_{\mathrm{ES}}^{\max } /$ Cap & 0.9 & 0.9 & 0.9 \\
$E_{\mathrm{ES}}^{\max } /$ Cap & 0.9 & 0.2 & 0.2 & 0.2 \\
$\gamma_{\mathrm{HS}}^{\max }$ & 0.2 & 0.2 & 0.2 & 0.2 \\
$\gamma_{\mathrm{HS} \text {, discharge }}^{\max }$ & 0.2 & 0.4 & 0.4 & 0.4 \\
$\eta_{\mathrm{HS} \text {,charge }}$ & 0.4 & 0.95 & 0.95 & 0.95 \\
$\eta_{\mathrm{HS} \text {,discharge }}$ & 0.95 & 0.95 & 0.95 & 0.95 \\
$\sigma_{\mathrm{HS}}$ & 0.95 & 0.1 & 0.1 & 0.1 \\
$E_{\mathrm{HS}}^{\max } /$ Cap & 0.1 & 0.9 & 0.9 & 0.1 \\
$E_{\mathrm{HS}}^{\max } /$ Cap & 0.9 & 0.1 & & \\
\hline & 0.1 & & 0.1 & \\
\hline & & & & \\
\hline
\end{tabular}

The time-of-use tariff is used for residential and industrial users in the example as shown in Appendix A (Table A1). Fixed tariffs are adopted for both commercial and office districts, both of which are $0.882 ¥ / \mathrm{kWh}$. IES sells electricity to the power grid at the price of $0.61 ¥ / \mathrm{kWh}$ and the converted natural gas at a calorific value of $0.29 ¥ / \mathrm{kWh}$, so $b_{\mathrm{h}}^{\mathrm{FDR}}=0.18 \mathrm{Y}=/(\mathrm{kWh})$. The maximum power point tracking mode is used for WT and PV. The real-time outputs of WT and PV are shown in Table A2. Electrical, thermal, and cooling loads in different districts are detailed in Tables A3 and A4.

This paper assumes that all four types of consumers are involved in the FDR project of electric load. The dispatching results are analyzed when the level of consumer's participation in the FDR project (the proportion of shiftable load occupying total electric load) is $5 \%, 10 \%$ and $20 \%$, respectively. Four types of users are involved in the FDR project of thermal load. Assuming that thermal load in the case is heating load, the outdoor temperature curve is shown in Figure A1. The temperature comfort ranges of heating load are constant $20^{\circ} \mathrm{C}, 20 \pm 1^{\circ} \mathrm{C}$, and $20 \pm 2{ }^{\circ} \mathrm{C}$, respectively.

\subsection{Simulation Results and Analysis}

\subsubsection{Benefit Analysis for FDR of Electric and Thermal Load}

As illustrated in Table 5, with the increase of consumer's participation in FDR, the total cost of the system operation will be reduced. The influence of the increase of shiftable load ratios and the 
temperature comfort range of heating load is significant. The total cost of $20 \%$ shiftable load ratio and $20 \pm 2{ }^{\circ} \mathrm{C}$ heating temperature comfort range is reduced by $4.58 \%$ compared with that of the scenario without considering FDR (The analysis below is based on this scenario without the special explanation). The total electric power consumption remains unchanged after the implementation of the FDR of electric load, but due to the load transfer, the valley period and the flat period with lower electricity prices or the period with more abundant electricity can be used to meet load demand, which reduces the cost of the electric load supply. After the implementation of the FDR of thermal load, the cost of thermal load supply is reduced by keeping the indoor temperature of each district within the comfort constraints, and timely regulating the thermal load supply.

Table 5. Costs of the system operation with FDR of electric and thermal load (Unit: $¥$ ).

\begin{tabular}{ccccc}
\hline \multirow{2}{*}{ FDR-Temperature Range of the Heating Load } & \multicolumn{4}{c}{ FDR-Ratio of the Shiftable Load } \\
\cline { 2 - 5 } & $\mathbf{0 \%}$ & $\mathbf{5 \%}$ & $\mathbf{1 0 \%}$ & $\mathbf{2 0} \%$ \\
\hline Constant $20^{\circ} \mathrm{C}$ & 239,060 & 237,280 & 235,230 & 231,550 \\
{$[19,21]^{\circ} \mathrm{C}$} & 237,390 & 235,610 & 223,530 & 229,780 \\
{$[18,22]{ }^{\circ} \mathrm{C}$} & 235,740 & 223,960 & 221,880 & 228,100 \\
\hline
\end{tabular}

Figure 6 shows the load transfer of residential and industrial districts. EFDR represents the transfer amount of electric load. Load-Eo represents the original electric load. Load-En represents the optimized electric load. The implementation of FDR of electric load effectively reduces the peak-valley difference of electric load in each district. Load in the period of high electricity price is transferred to the periods of high wind power generation or low electricity price, which plays the role of reducing peak and filling valley.
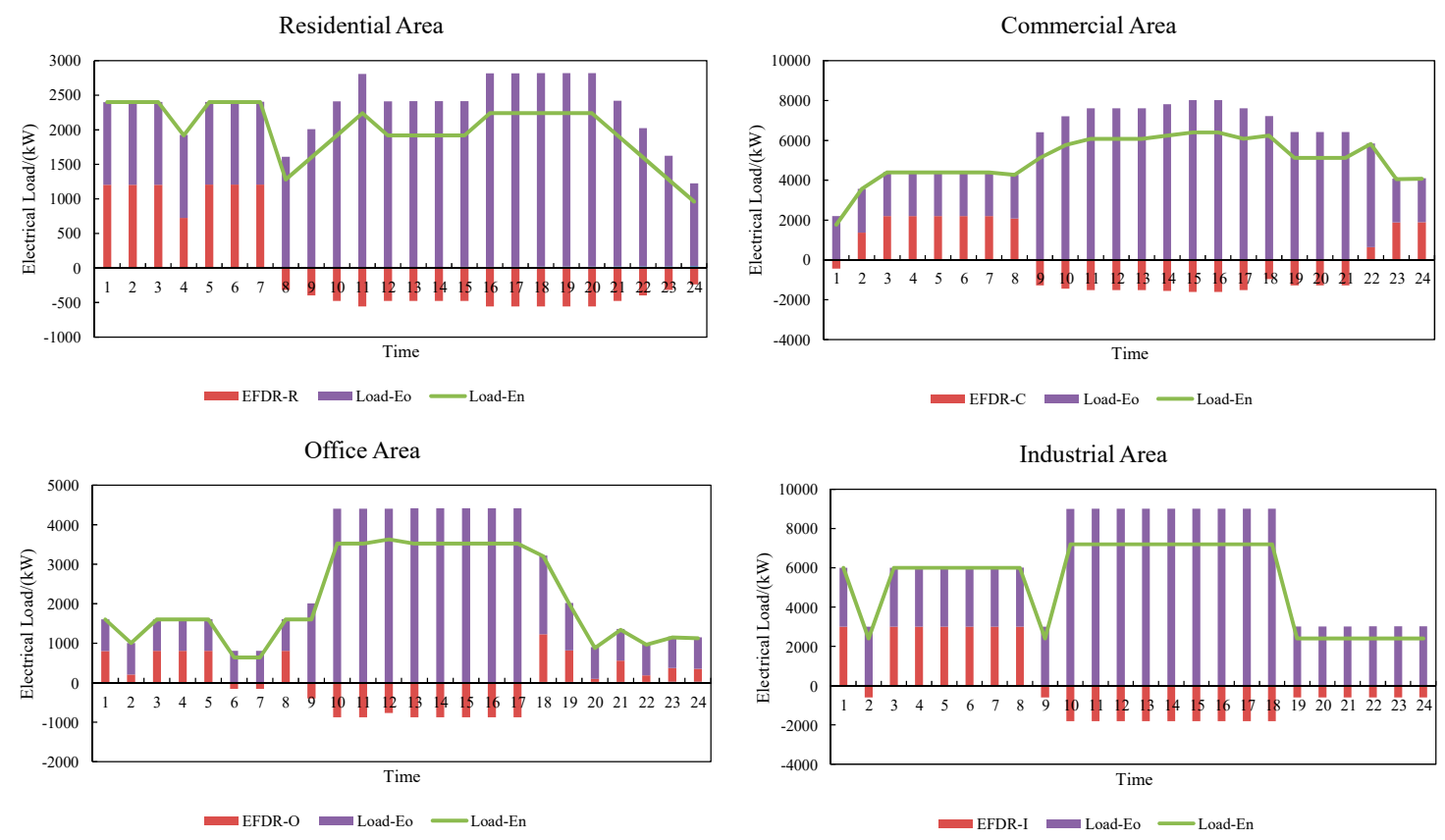

Figure 6. Shift situation of electrical load after FDR of electric load.

Figure 7 shows the indoor temperature in each district. It can be seen that the indoor temperature after optimization is satisfied with the constraints of consumer's comfort, and the indoor temperature is maintained in the range of $20 \pm 2{ }^{\circ} \mathrm{C}$. Figure 8 shows the situation of thermal load regulation. HFDR represents the heating load regulation. Load-Ho represents the original heating load. Load-Hn represents the optimized heating load. The implementation of FDR of thermal load reduces the 
heating load supply and directly reduces the total operating cost under the condition of satisfying the consumer's comfort and providing economic compensation.

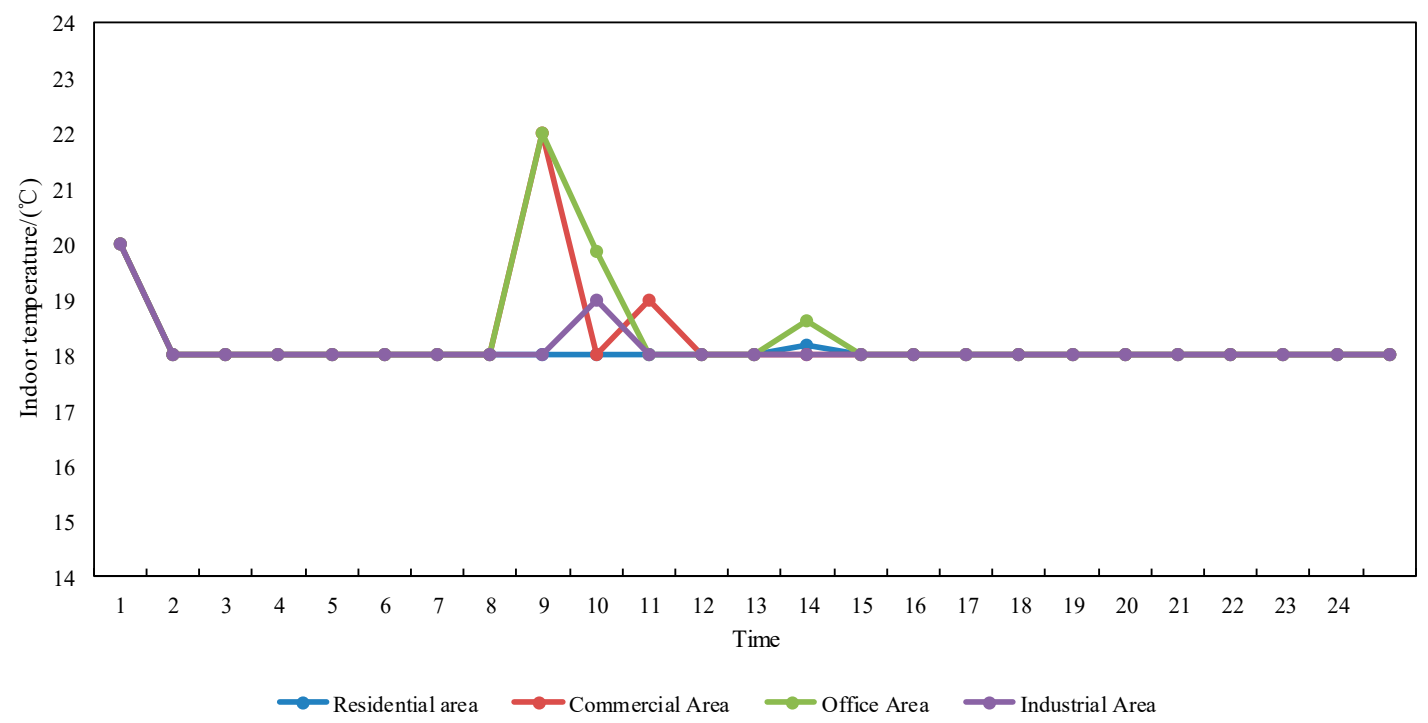

Figure 7. Indoor temperature of multi-district IES.
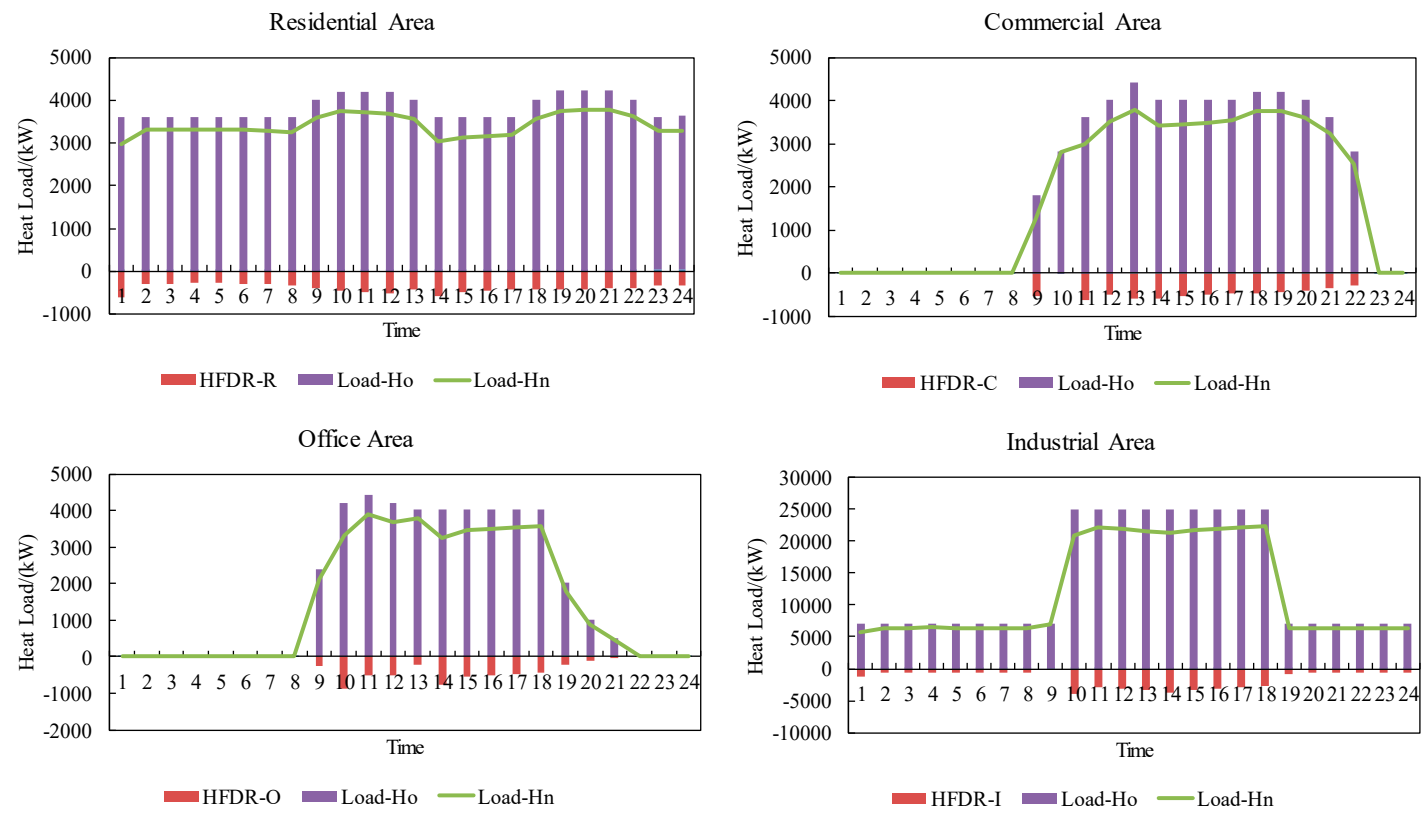

Figure 8. Adjustment situation of heating load after FDR of thermal load.

\subsubsection{Operation Analysis for Multi-District IES}

The optimization results of electric power and thermal power in the system are shown in Figures 9 and 10, respectively. The positive power output of the power grid is to indicate that the system purchases electricity from the power grid, while negative power to indicate that the system sells electricity to the power grid. The output of ES is positive to indicate that ES discharges, while negative to indicate that ES charges. The output of HS is positive to indicate that HS releases heat, and negative to indicate that HS stores heat. GT in residential district mainly works at 11:00-15:00 and 18:00-21:00, and the electricity in the remaining periods is mainly purchased from the power grid. The electricity demand in commercial and office districts is mainly met by GT, WT, and PV. There is no need to buy electricity from the grid, and electricity will be sold to the grid when the power is surplus. Power 
supply in industrial district is mainly met by the power grid, GT, WT, and PV, but GT mainly works at the peak of power grid (8:00-24:00) and sells surplus power to the grid.
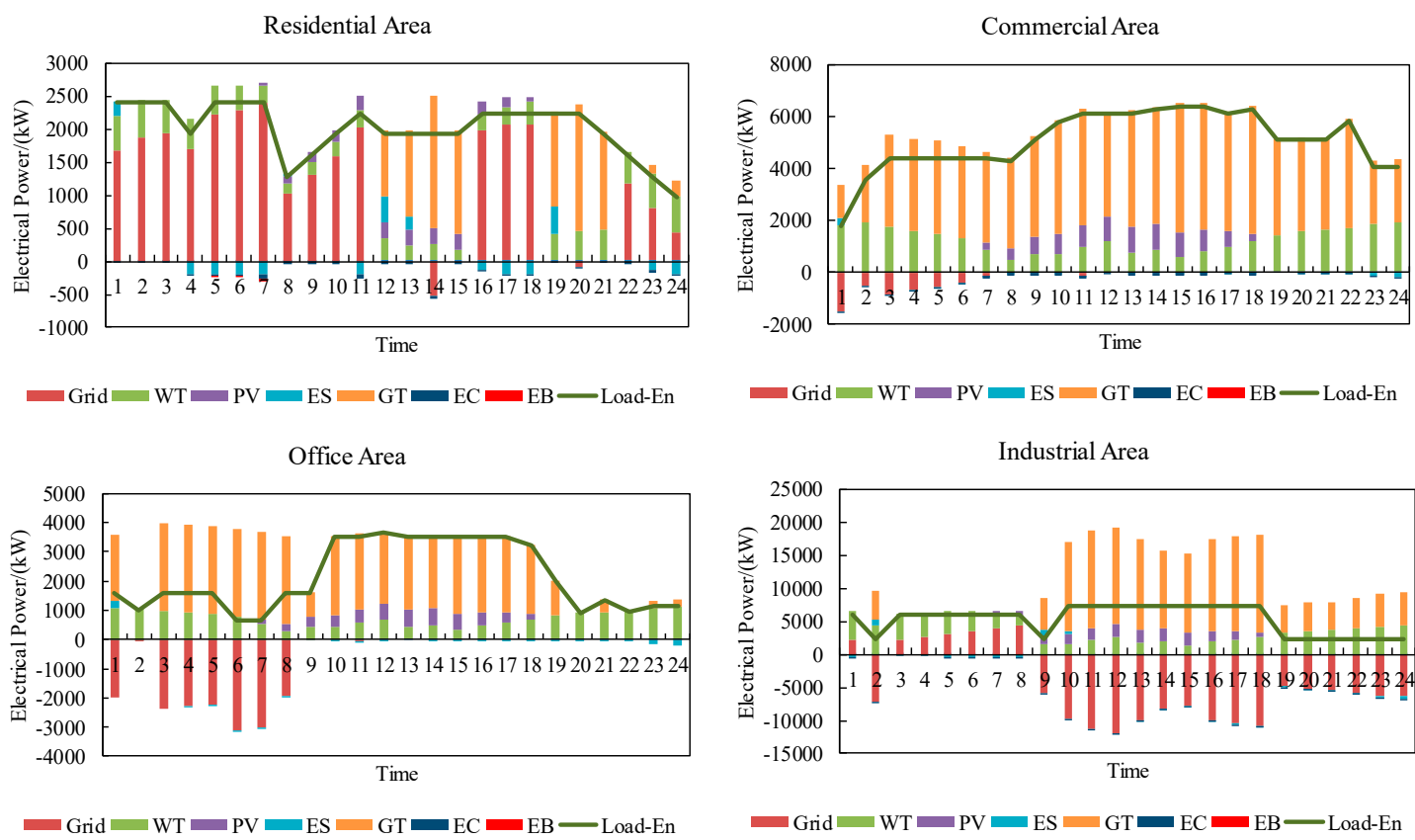

Figure 9. Dispatching results of electric power.
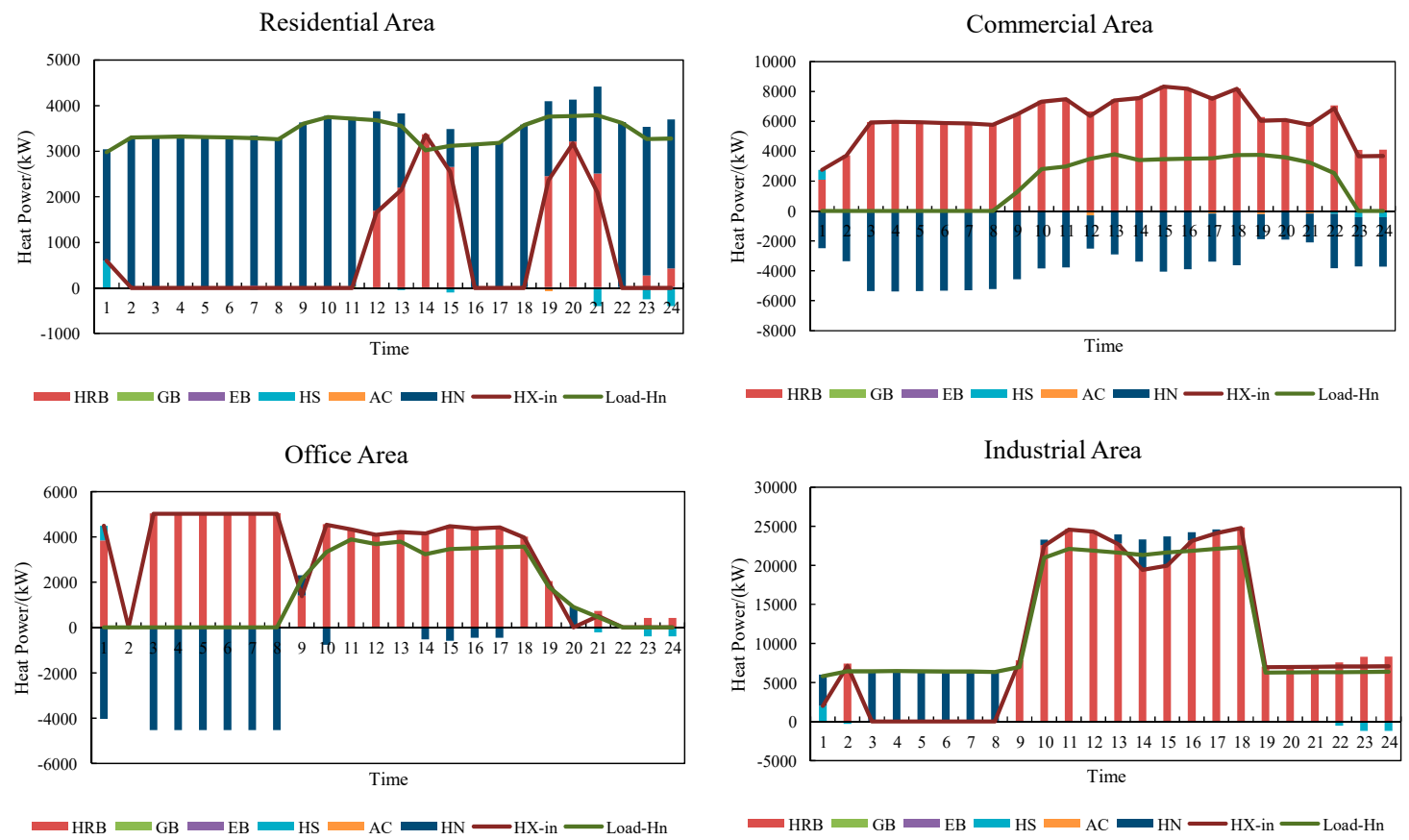

Figure 10. Dispatching results of heating power.

DHN denotes the interactive heating power between each district of multi-district IES and the heating network. The positive value denotes the heating power obtained from the heating network, and negative denotes the heating power injected into the heating network. As can be seen from Figure 10, most of the heating energy supply in residential district is obtained by the heating network, and GT mainly produces heating energy at 11:00-15:00 and 18:00-21:00. Industrial district also obtains heating energy from the heating network, but most of its heating energy is generated by its GT. Obviously, the load of residential and industrial districts is relatively high, so more heating energy is obtained from 
the heating network. The load of commercial and office areas is relatively low. During the operation, the surplus heat energy of them is transferred to residential and industrial districts through the heating network. It can be seen that the district heating network achieves the coordinated distribution of heating energy among different districts, further improves the efficiency of heating energy utilization, and reduces the operating cost of the system.

\subsubsection{Analysis of Heating Network Condition and Benefit}

(1) Operation conditions of the heating network

The velocity and power of heating medium in the heating network are shown in Figure 11. The value of clockwise flow of the heating medium is positive, and that of counter-clockwise flow is negative. According to Figure 11, it can be seen that the curve trend of velocity of heating medium and heating power is the same, which satisfies the actual operation constraints of the heating network. It can be concluded that: IES-1 mainly obtains thermal energy from IES-2, and only at 12:00-13:00, IES-4 provides thermal energy for IES-1. IES-2 mainly acts as a thermal energy transporter, conveying thermal energy outward through pipelines 1-2 and 2-3. IES-3 mainly acts as a thermal energy transporter, conveying heat energy to IES-4 through pipelines 3-4. IES-4 only obtains thermal energy from IES-3 during the whole operation cycle. These conclusions are consistent with Figure 10.
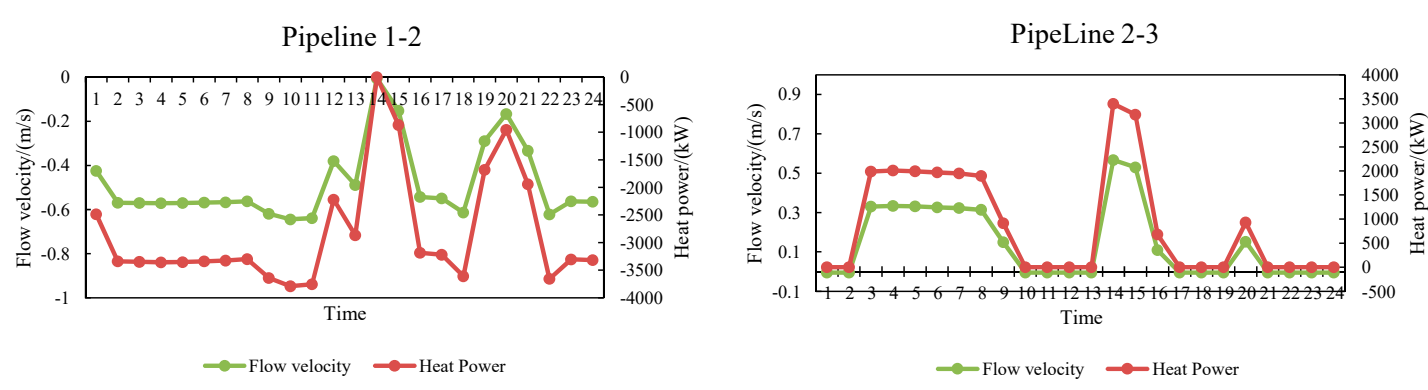

Pipeline 3-4
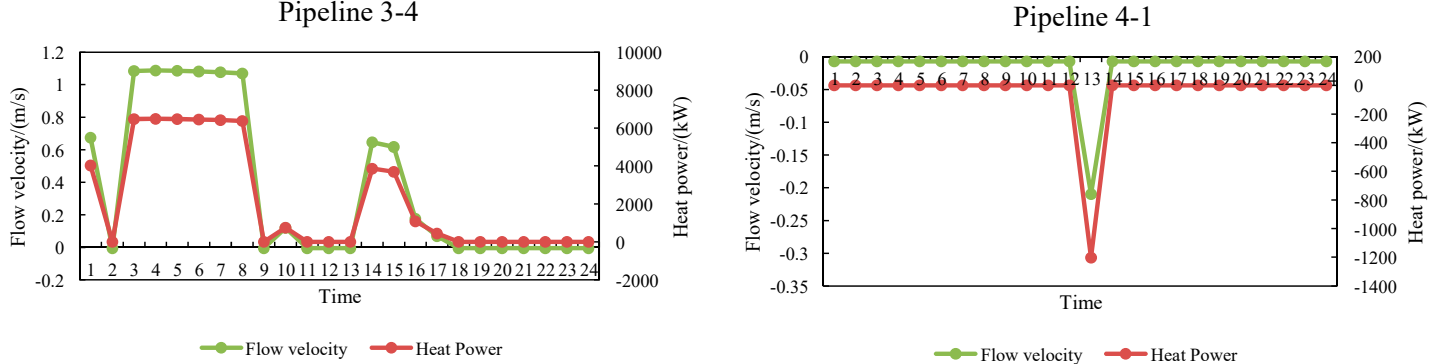

Figure 11. Flow velocity and thermal power of the heating medium in the heating network.

(2) Benefit analysis of the heating network

This paper makes a comparative analysis of the heating network operating benefits, ES and HS. The results are shown in Table 6. Comparing case 1 with case 5, it can be seen that when the system does not contain the heating network, the cost of the purchasing electricity increases significantly. The compensation cost of FDR for thermal load and the cost of natural gas decreases slightly. The daily operation cost of multi-district IES with the heating network decreases by $8.52 \%$ compared with that without the heating network. The existence of the heating network will directly affect the cost of purchasing electricity and the cost of FDR compensation for thermal load. The reason is that the heating network provides a direct energy coupling exchange channel for districts in the IES, redistributes the heat-to-electric ratios among districts, and reduces the total cost of the IES operation. In addition, comparing case 1 with cases $2 \sim 4$, and case 5 with cases $6 \sim 8$, it can be found that the total operating cost increases when the IES is equipped with ES and HS. Considering that the role of the heating network has met the optimal operating requirements of the system in the case, the addition of energy storage devices is needless. Their self-consumption rates lead to the reaction of the increasing cost after 
joining. The total operating cost of case 1 (without considering the self-consumption rates) is $226,190 ¥$, which is $490 ¥$ lower than that of case 4 . The total operating cost of case 5 (without considering the self-consumption rates) is $247,140 ¥$, which is $940 ¥$ lower than that of case 8 . This further verifies that the role of energy storage devices in this example is not obvious, and its own electric and thermal loss increases the total operating cost. Therefore, the number and capacities of energy storage devices must be reasonably set up in the actual planning process of multi-district IES with heating networks to ensure the operation economy.

Table 6. Comparison of operating benefits among the district heating network, ES, and HS (Unit: ¥).

\begin{tabular}{cccccccccc}
\hline Case & DHN & ES & HS & $\mathbf{C}_{\mathbf{e}, \mathbf{b}}$ & $\mathbf{C}_{\mathbf{e}, \mathbf{s}}$ & $\mathbf{C}_{\text {gas }}$ & $\mathbf{C}_{\mathbf{H N}}$ & $\mathbf{C}_{\mathbf{h}}{ }^{\text {FDR }}$ & $\mathbf{C}_{\text {MD-IES }}$ \\
\hline 1 & $\sqrt{ }$ & $\sqrt{ }$ & $\sqrt{ }$ & 18,949 & 97,660 & 295,650 & 395 & 10,770 & 228,100 \\
2 & $\sqrt{ }$ & $\sqrt{ }$ & $\times$ & 19,114 & 96,497 & 293,290 & 404 & 10,778 & 227,080 \\
3 & $\sqrt{ }$ & $\times$ & $\sqrt{ }$ & 17,539 & 96,380 & 295,330 & 411 & 10,798 & 227,700 \\
4 & $\sqrt{ }$ & $\times$ & $\times$ & 17,690 & 95,235 & 293,060 & 419 & 10,752 & 226,680 \\
5 & $\times$ & $\sqrt{ }$ & $\sqrt{ }$ & 54,185 & 99,147 & 285,690 & 0 & 8625 & 249,350 \\
6 & $\times$ & $\times$ & $\sqrt{ }$ & 51,899 & 99,392 & 287,550 & 0 & 8626 & 248,680 \\
7 & $\times$ & $\sqrt{ }$ & $\times$ & 56,307 & 97,574 & 281,390 & 0 & 8625 & 248,750 \\
8 & $\times$ & $\times$ & $\times$ & 55,418 & 97,355 & 281,390 & 0 & 8625 & 248,080 \\
\hline
\end{tabular}

\subsubsection{Analysis in Economy}

(1) Sensitivity analysis of the real-time compensation price of thermal load

In this paper, the sensitivity analysis of the real-time compensation price for thermal load is carried out. The result is shown in Figure 12. Without considering the real-time compensation of FDR for thermal load, the total operating cost of the system can be reduced to $216,825 ¥$, which is $6.36 \%$ lower than that of $b_{\mathrm{h}}^{\mathrm{FDR}}>0.26 \mathrm{Y}=/(\mathrm{kWh})$. With the increase of the real-time compensation price, the total operating cost is also increasing, which is approximately linear. When $b_{\mathrm{h}}^{\mathrm{FDR}}>0.26 \mathrm{Y}=/(\mathrm{kWh})$, the cost is approximately maintained at $231,547 ¥$. According to the calculation example, it is found that thermal load no longer participates in FDR. This is because the compensation price is close to the calorific value price of natural gas. Considering the loss of thermal power flowing in the system, the system will not excavate the benefit of HDR for thermal load. The system directly meets the heating load supply.

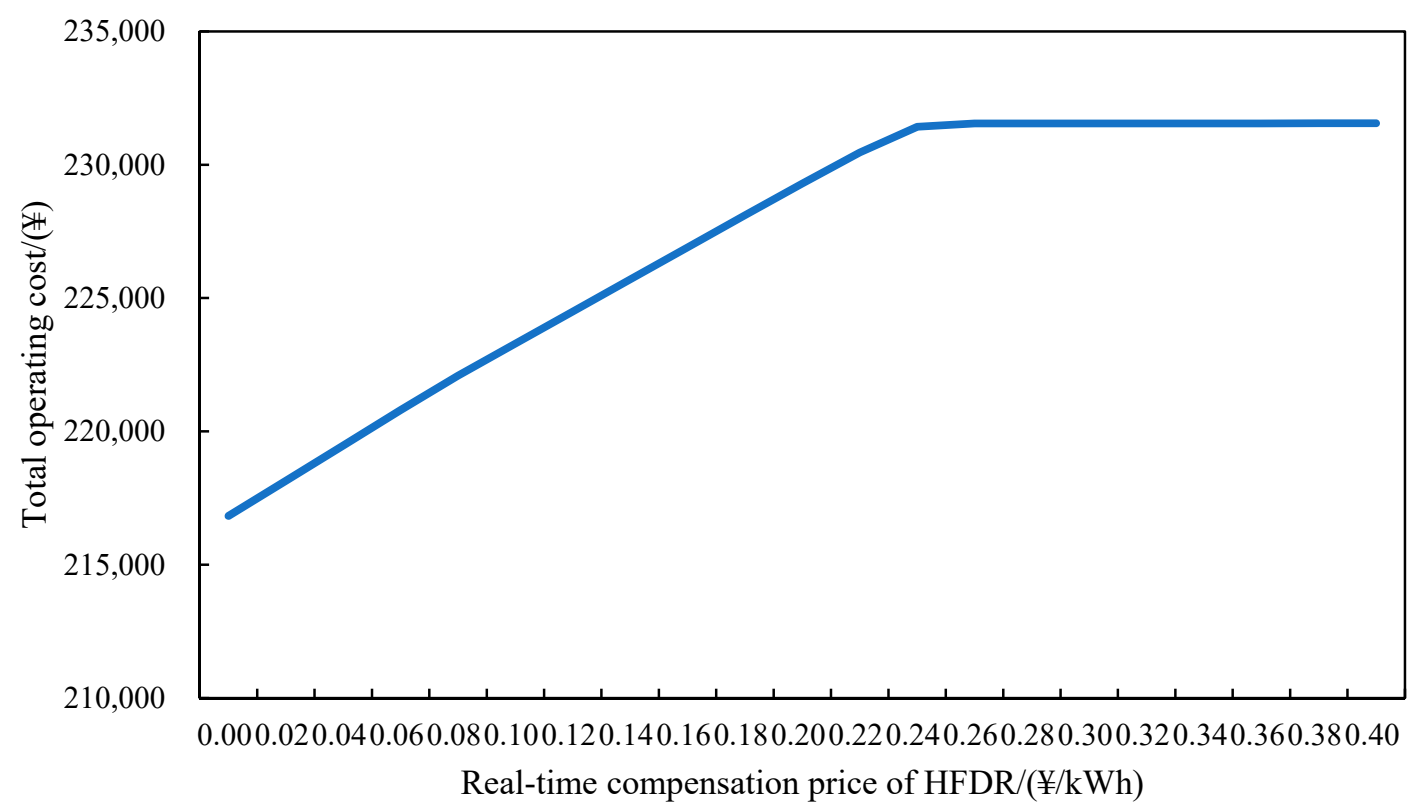

Figure 12. Sensitivity analysis of the real-time compensation price of thermal load. 
(2) Sensitivity analysis of the calorific value price of natural gas

Figure 13 shows the relationships among the operating cost, the difference of operating cost (the difference between the total operating cost of the system without heating network and the total operating cost of the system with heating network) and the price of natural gas under different electricity prices. With or without the heating network, the total operating cost increases with the calorific value price of natural gas. The difference of the operating cost shows a downward trend. The total operating cost of the system taking different electricity selling prices also shows a similar trend with the increase of the calorific value price of natural gas. It can be seen that when $c_{\text {gas }}$ is greater than a certain critical value, the operating conditions with and without heating network tend to be consistent.
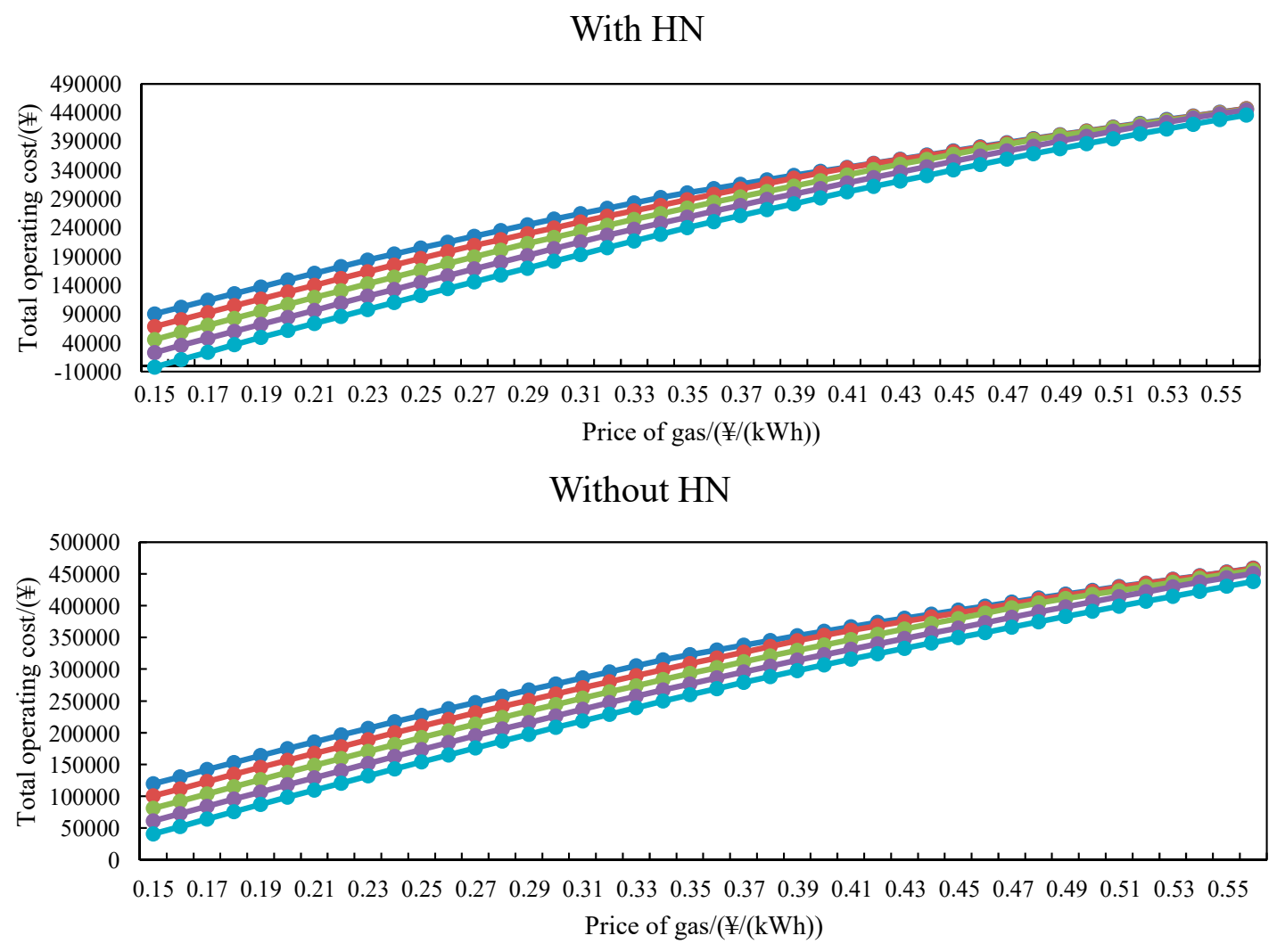

Difference of costs

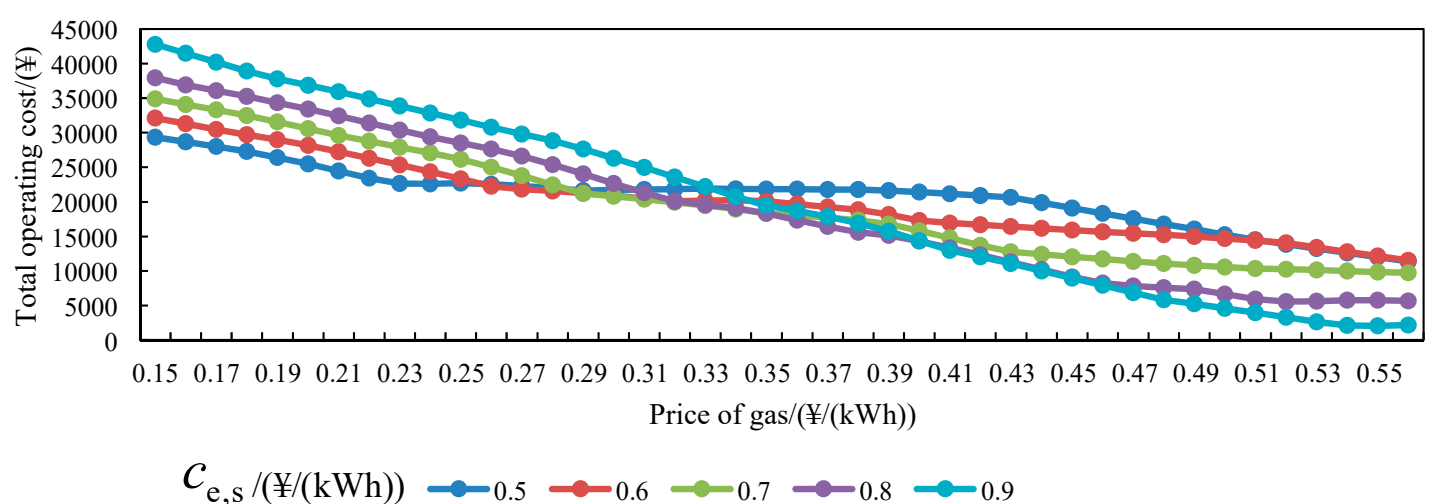

Figure 13. Sensitivity analysis of the total operating cost and the price of natural gas.

Tables 7 and 8 give the operating costs of different calorific value prices of natural gas with and without heating networks, respectively when $c_{\mathrm{e}, \mathrm{s}}=0.7 ¥ / \mathrm{kWh}$. It can be seen that when $c_{\text {gas }}$ is low, 
the coupling of multi-district load (mainly heating load) makes its economic benefit more remarkable. Essentially, by transferring heating load, the heat-to-electric ratios of load in some districts is increased, while the heat-to-electric ratios of load in other districts is reduced. GT in the district with the high heat-to-electric ratio of load can bring greater economic benefit. With the increase of $c_{\text {gas }}$, the electricity purchased from the power grid shows an upward trend, and the electricity sold to the power grid is decreasing. The system tends to consume more electricity to meet the load demand. GT tends to produce less heat abandonment and make full use of its heat energy. In addition, the system is more inclined to meet thermal load demand directly when $c_{\text {gas }}$ is lower. When $c_{\text {gas }}$ increase to a critical value, the system will excavate FDR resources of thermal load.

Table 7. Relation between the total operating cost and the price of natural gas with the heating network when $\mathrm{c}_{\mathrm{e}, \mathrm{s}}=0.7 ¥ / \mathrm{kWh}$.

\begin{tabular}{ccccccc}
\hline $\mathbf{C}_{\text {gas }} /(¥ / \mathbf{k W h})$ & $\mathbf{C}_{\mathbf{e}, \mathbf{b}} /(¥)$ & $\mathbf{C}_{\mathbf{e}, \mathbf{s}} /(¥)$ & $\mathbf{C}_{\text {gas }} /(¥)$ & $\mathbf{C}_{\text {HN }} /(¥)$ & $\mathbf{C}_{\mathbf{h}}{ }^{\text {FDR }} /(¥)$ & $\mathbf{C}_{\text {MD-IES }} /(¥)$ \\
\hline 0.15 & 18,462 & 155,510 & 182,400 & 580 & 0 & 45,929 \\
0.25 & 18,959 & 147,570 & 294,150 & 509 & 0 & 166,050 \\
0.35 & 26,063 & 101,270 & 338,720 & 395 & 10,823 & 274,730 \\
0.45 & 47,152 & 783,01 & 387,500 & 519 & 10,831 & 367,700 \\
0.55 & 77,609 & 6950 & 356,310 & 684 & 11,746 & 439,400 \\
\hline
\end{tabular}

Table 8. Relation between the total operating cost and the price of natural gas without the heating network when $\mathrm{C}_{\mathrm{e}, \mathrm{s}}=0.7 ¥ / \mathrm{kWh}$.

\begin{tabular}{ccccccc}
\hline $\mathbf{C}_{\text {gas }} /(¥ / \mathbf{k W h})$ & $\mathbf{C}_{\mathbf{e}, \mathbf{b}} /(¥)$ & $\mathbf{C}_{\mathbf{e}, \mathbf{s}} /(¥)$ & $\mathbf{C}_{\text {gas }} /(¥)$ & $\mathbf{C}_{\text {HN }} /(¥)$ & $\mathbf{C}_{\mathbf{h}}{ }^{\text {FDR }} /(¥)$ & $\mathbf{C}_{\text {MD-IES} /(¥)}$ \\
\hline 0.15 & 40,201 & 135,960 & 170,170 & 0 & 6427 & 80,834 \\
0.25 & 44,804 & 130,120 & 271,480 & 0 & 6055 & 192,220 \\
0.35 & 69,089 & 112,270 & 325,450 & 0 & 10,780 & 293,050 \\
0.45 & 99,891 & 102,290 & 371,370 & 0 & 10,805 & 379,770 \\
0.55 & 123,260 & 34,882 & 349,160 & 0 & 11,720 & 449,260 \\
\hline
\end{tabular}

(3) Sensitivity analysis of the heat-to-electric ratio of GT

The influence of the heat-to-electric ratio of GT on the operation economy of IES-2 (commercial consumer) and IES-4 (industrial consumer) with high heating load is analyzed. By changing the heat-to-electric ratio of GT in these two districts, the relationship curve between the total operating cost and the heat-to-electric ratio can be obtained, as shown in Figure 14. For IES-2 with the lower heat-to-electric ratio of load and IES-4 with the higher heat-to-electric ratio of load, increasing the heat-to-electric ratio of GT can reduce the total operating cost. This is due to the high proportion of heating load in the example. After the heat-to-electric ratio of GT is increased, only less gas consumption is needed to meet the heating load demand, which directly reduces the gas consumption. According to Table 5, the gas cost occupies the main part of the total cost. Obviously, when IES operates independently in a single district, the change of the heat-to-electric ratio of GT has little effect on the electric and thermal power scheduling in the system, but for multi-district IES with the heating network, the change of the heat-to-electric ratio of GT in any district will cause the redistribution of heating load, and the district with higher heat-to-electric ratio will produce more thermal power. 


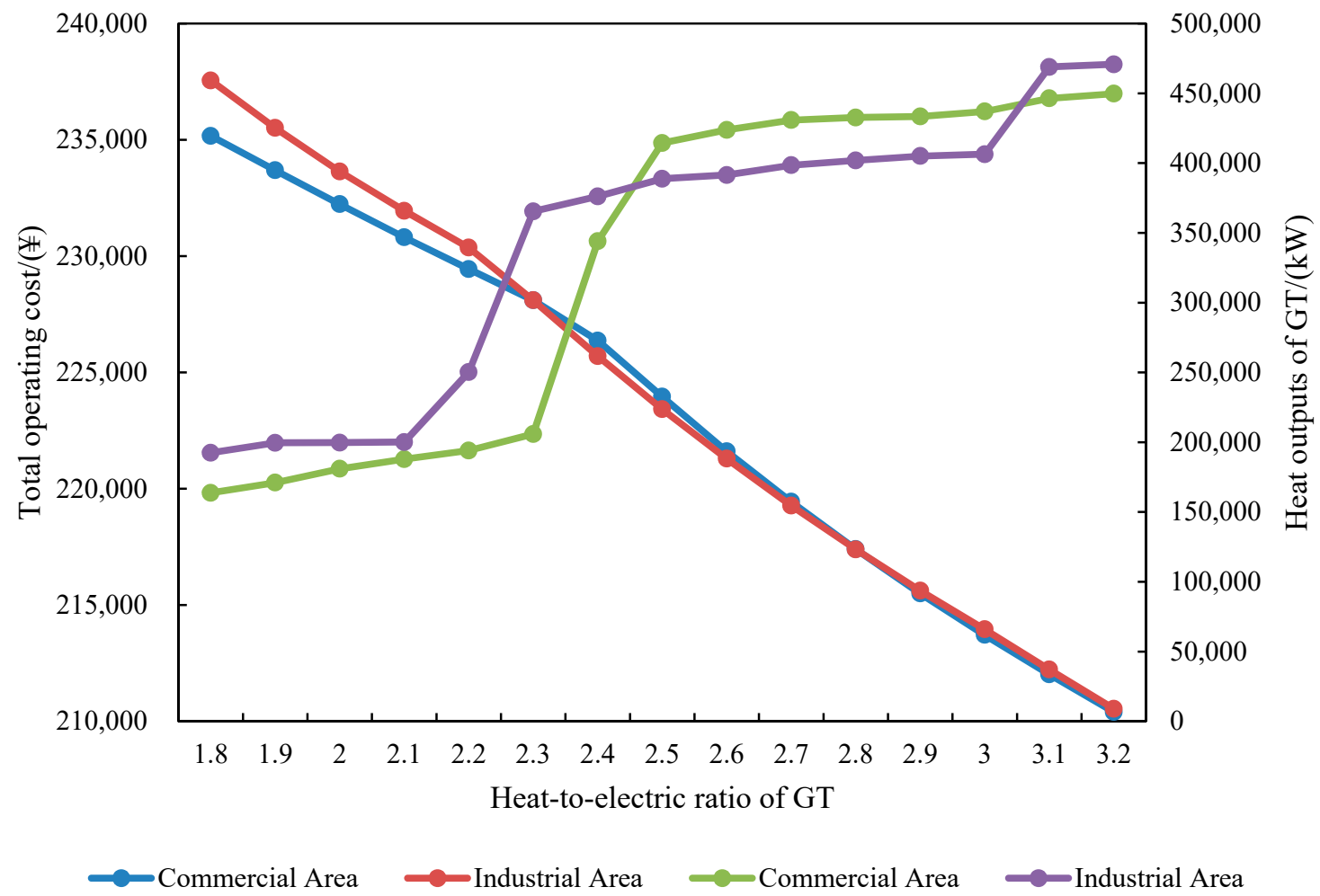

Figure 14. Sensitivity analysis of the total operating cost and the heat-to-electric ratio of GT.

\section{Conclusions}

Based on the comprehensive consideration of FDR of electric and thermal load, the IES energy coupling structure and operation characteristics of heating networks, a multi-district IES operation optimization model considering FDR of electric and thermal load is proposed. Through the case simulation and theoretical study, the following conclusions can be drawn:

(1) The FDR control strategy of electric and thermal loads is introduced. On the premise of meeting the demand of electric and thermal loads in different IES districts, the elasticity and flexibility of supply side and demand side are increased by transferring electric load, reducing real-time thermal load. The peak-valley difference of electric and thermal load, and the total operating cost of the system are effectively reduced.

(2) The optimal operation model of multi-district IES with the DHN is proposed. On the one hand, it can realize the optimal operation condition of the equipment in each district and achieve the optimal work coordination. On the other hand, the load characteristics of different districts can be coupled through the DHN. The complementary characteristics can be used to optimize the energy flow and improve the operation economy.

(3) The application of the DHN in multi-district IES with large electric and thermal load and uneven distribution of heat-to-electric ratios among sub-districts is more significant. By connecting CCHP systems in different districts through the DHN, the coordinated distribution of energy among sub-districts can be realized. The utilization efficiency of thermal energy can also be improved. The role of the DHN is essentially to transfer heating load to redistribute the heat-to-electric ratios to achieve the optimal operation of the system. Based on the example in this paper, the interconnection of DHN also plays the role of substituting ES and HS in each sub-district, which can reduce the investment cost of energy storage devices in the planning and construction. 
Author Contributions: The methodology was proposed by C.Z., J.Z., and S.L., the investigation was done by C.Z. and S.L., the formal analysis work was done by Y.L. and F.M., the validation work was done by Y.P., the data curation work was finished by T.S. and J.W., and the draft was finished by C.Z. and S.L.

Funding: This research was funded by National Key R\&D Program of China (2018YFB0905002).

Conflicts of Interest: The authors declare no conflict of interest.

\section{Appendix A}

Table A1. Time-of-use electricity price.

\begin{tabular}{cccc}
\hline Area & Category & Concrete Time & Price/(¥/kWh) \\
\hline Resident & \multirow{2}{*}{ Peak period } & $12: 00 \sim 16: 00,19: 00 \sim 22: 00$ & 0.89 \\
Industry & & $9: 00 \sim 13: 00,18: 00 \sim 22: 00$ & 1.435 \\
\hline Resident & \multirow{2}{*}{ Normal period } & $8: 00 \sim 12: 00,16: 00 \sim 19: 00,22: 00 \sim 24: 00$ & 0.49 \\
Industry & & $13: 00 \sim 18: 00,22: 00 \sim 24: 00$ & 0.861 \\
\hline Resident & \multirow{2}{*}{ Valley period } & $0: 00 \sim 8: 00$ & 0.25 \\
Industry & & $0: 00 \sim 9: 00$ & 0.387 \\
\hline
\end{tabular}

Table A2. Forecast outputs of WT and PV.

\begin{tabular}{|c|c|c|c|c|c|c|c|c|}
\hline \multirow{2}{*}{ Time } & \multicolumn{4}{|c|}{ Forecast Outputs of $\mathrm{WT} /(\mathbf{k W})$} & \multicolumn{4}{|c|}{ Forecast Outputs of PV/(kW) } \\
\hline & Resident & Commerce & Office & Industry & Resident & Commerce & Office & Industry \\
\hline 00:00 01:00 & 525 & 1837.5 & 1050 & 4200 & 0 & 0 & 0 & 0 \\
\hline 01:00 02:00 & 545 & 1907.5 & 1090 & 4360 & 0 & 0 & 0 & 0 \\
\hline 02:00 03:00 & 495 & 1732.5 & 990 & 3960 & 0 & 0 & 0 & 0 \\
\hline 03:00 04:00 & 450 & 1575 & 900 & 3600 & 0 & 0 & 0 & 0 \\
\hline 04:00 05:00 & 427.5 & 1496.25 & 855 & 3420 & 0 & 0 & 0 & 0 \\
\hline 05:00 06:00 & 375 & 1312.5 & 750 & 3000 & 0 & 0 & 0 & 0 \\
\hline 06:00 07:00 & 255 & 892.5 & 510 & 2040 & 61.8 & 255 & 139.2 & 507.6 \\
\hline 07:00 08:00 & 137.5 & 481.25 & 275 & 1100 & 110.4 & 458.4 & 252 & 914.4 \\
\hline 08:00 09:00 & 200 & 700 & 400 & 1600 & 160.2 & 661.8 & 363 & 1320 \\
\hline 09:00 10:00 & 200 & 700 & 400 & 1600 & 184.8 & 763.2 & 417 & 1523.4 \\
\hline $10: 00 \sim 11: 00$ & 275 & 962.5 & 550 & 2200 & 209.4 & 865.8 & 474 & 1726.8 \\
\hline $11: 00 \sim 12: 00$ & 335 & 1172.5 & 670 & 2680 & 234 & 966 & 531 & 1932 \\
\hline $12: 00 \sim 13: 00$ & 220 & 770 & 440 & 1760 & 246 & 990 & 558 & 2032.2 \\
\hline $13: 00 \sim 14: 00$ & 242.5 & 848.75 & 485 & 1940 & 246 & 990 & 558 & 2032.2 \\
\hline $14: 00 \sim 15: 00$ & 162.5 & 568.75 & 325 & 1300 & 234 & 966 & 528 & 1930.2 \\
\hline $15: 00 \sim 16: 00$ & 232.5 & 813.75 & 465 & 1860 & 196.8 & 814.8 & 447 & 1625.4 \\
\hline $16: 00 \sim 17: 00$ & 275 & 962.5 & 550 & 2200 & 147.6 & 611.4 & 336 & 1219.2 \\
\hline $17: 00 \sim 18: 00$ & 335 & 1172.5 & 670 & 2680 & 73.8 & 306 & 168 & 609.6 \\
\hline $18: 00 \sim 19: 00$ & 397.5 & 1391.25 & 795 & 3180 & 0 & 0 & 0 & 0 \\
\hline 19:00 20:00 & 442.5 & 1548.75 & 885 & 3540 & 0 & 0 & 0 & 0 \\
\hline $20: 00 \sim 21: 00$ & 460 & 1610 & 920 & 3680 & 0 & 0 & 0 & 0 \\
\hline $21: 00 \sim 22: 00$ & 485 & 1697.5 & 970 & 3880 & 0 & 0 & 0 & 0 \\
\hline $22: 00 \sim 23: 00$ & 522.5 & 1828.75 & 1045 & 4180 & 0 & 0 & 0 & 0 \\
\hline $23: 00 \sim 24: 00$ & 542.5 & 1898.75 & 1085 & 4340 & 0 & 0 & 0 & 0 \\
\hline
\end{tabular}


Table A3. Electric and thermal load.

\begin{tabular}{|c|c|c|c|c|c|c|c|c|}
\hline \multirow{2}{*}{ Time } & \multicolumn{4}{|c|}{ Electric load/(kW) } & \multicolumn{4}{|c|}{ Thermal Load/(kW) } \\
\hline & Resident & Commerce & Office & Industry & Resident & Commerce & Office & Industry \\
\hline 00:00 01:00 & 1200 & 2200 & 800 & 3000 & 3600 & 0 & 0 & 7000 \\
\hline 01:00 02:00 & 1200 & 2200 & 800 & 3000 & 3600 & 0 & 0 & 7000 \\
\hline 02:00 03:00 & 1200 & 2200 & 800 & 3000 & 3600 & 0 & 0 & 7000 \\
\hline 03:00 04:00 & 1200 & 2200 & 800 & 3000 & 3600 & 0 & 0 & 7000 \\
\hline 04:00 05:00 & 1200 & 2200 & 800 & 3000 & 3600 & 0 & 0 & 7000 \\
\hline 05:00 06:00 & 1200 & 2200 & 800 & 3000 & 3600 & 0 & 0 & 7000 \\
\hline 06:00 07:00 & 1200 & 2200 & 800 & 3000 & 3600 & 0 & 0 & 7000 \\
\hline 07:00 08:00 & 1600 & 2200 & 800 & 3000 & 3600 & 0 & 0 & 7000 \\
\hline 08:00 09:00 & 2000 & 6400 & 2000 & 3000 & 4000 & 1800 & 2400 & 7000 \\
\hline 09:00 10:00 & 2400 & 7200 & 4400 & 9000 & 4200 & 2800 & 4200 & 25,000 \\
\hline 10:00 11:00 & 2800 & 7600 & 4400 & 9000 & 4200 & 3600 & 4400 & 25,000 \\
\hline $11: 00 \sim 12: 00$ & 2400 & 7600 & 4400 & 9000 & 4200 & 4000 & 4200 & 25,000 \\
\hline $12: 00 \sim 13: 00$ & 2400 & 7600 & 4400 & 9000 & 4000 & 4400 & 4000 & 25,000 \\
\hline 13:00 14:00 & 2400 & 7800 & 4400 & 9000 & 3600 & 4000 & 4000 & 25,000 \\
\hline $14: 00 \sim 15: 00$ & 2400 & 8000 & 4400 & 9000 & 3600 & 4000 & 4000 & 25,000 \\
\hline $15: 00 \sim 16: 00$ & 2800 & 8000 & 4400 & 9000 & 3600 & 4000 & 4000 & 25,000 \\
\hline $16: 00 \sim 17: 00$ & 2800 & 7600 & 4400 & 9000 & 3600 & 4000 & 4000 & 25,000 \\
\hline 17:00 18:00 & 2800 & 7200 & 2000 & 9000 & 4000 & 4200 & 4000 & 25,000 \\
\hline 18:00 19:00 & 2800 & 6400 & 1200 & 3000 & 4200 & 4200 & 2000 & 7000 \\
\hline 19:00 20:00 & 2800 & 6400 & 800 & 3000 & 4200 & 4000 & 1000 & 7000 \\
\hline 20:00 21:00 & 2400 & 6400 & 800 & 3000 & 4200 & 3600 & 500 & 7000 \\
\hline 21:00 22:00 & 2000 & 5200 & 800 & 3000 & 4000 & 2800 & 0 & 7000 \\
\hline $22: 00 \sim 23: 00$ & 1600 & 2200 & 800 & 3000 & 3600 & 0 & 0 & 7000 \\
\hline 23:00 24:00 & 1200 & 2200 & 800 & 3000 & 3600 & 0 & 0 & 7000 \\
\hline
\end{tabular}

Table A4. Cooling load.

\begin{tabular}{ccccc}
\hline \multirow{2}{*}{ Time } & \multicolumn{4}{c}{ Cooling Load/(kW) } \\
\cline { 2 - 5 } & Resident & Commerce & Office & Industry \\
\hline 00:00 01:00 & 100 & 230 & 0 & 290 \\
01:00 02:00 & 100 & 230 & 0 & 290 \\
02:00 03:00 & 100 & 230 & 0 & 290 \\
03:00 04:00 & 100 & 230 & 0 & 290 \\
04:00 05:00 & 100 & 230 & 0 & 290 \\
05:00 06:00 & 100 & 230 & 0 & 290 \\
06:00 07:00 & 200 & 410 & 0 & 290 \\
07:00 08:00 & 200 & 410 & 0 & 290 \\
08:00 09:00 & 200 & 410 & 0 & 290 \\
09:00 10:00 & 200 & 410 & 0 & 290 \\
10:00 11:00 & 200 & 410 & 0 & 290 \\
11:00 12:00 & 200 & 410 & 0 & 290 \\
12:00 13:00 & 200 & 410 & 0 & 290 \\
13:00 14:00 & 200 & 410 & 0 & 290 \\
14:00 15:00 & 200 & 410 & 0 & 290 \\
15:00 16:00 & 100 & 410 & 0 & 290 \\
16:00 17:00 & 100 & 410 & 0 & 290 \\
17:00 18:00 & 100 & 410 & 0 & 290 \\
18:00 19:00 & 100 & 230 & 0 & 290 \\
19:00 20:00 & 100 & 230 & 0 & 290 \\
20:00 21:00 & 100 & 230 & 0 & 290 \\
21:00 22:00 & 100 & 230 & 0 & 290 \\
22:00 23:00 & 100 & 230 & 0 & 290 \\
23:00 24:00 & 100 & 230 & 0 & \\
\hline
\end{tabular}




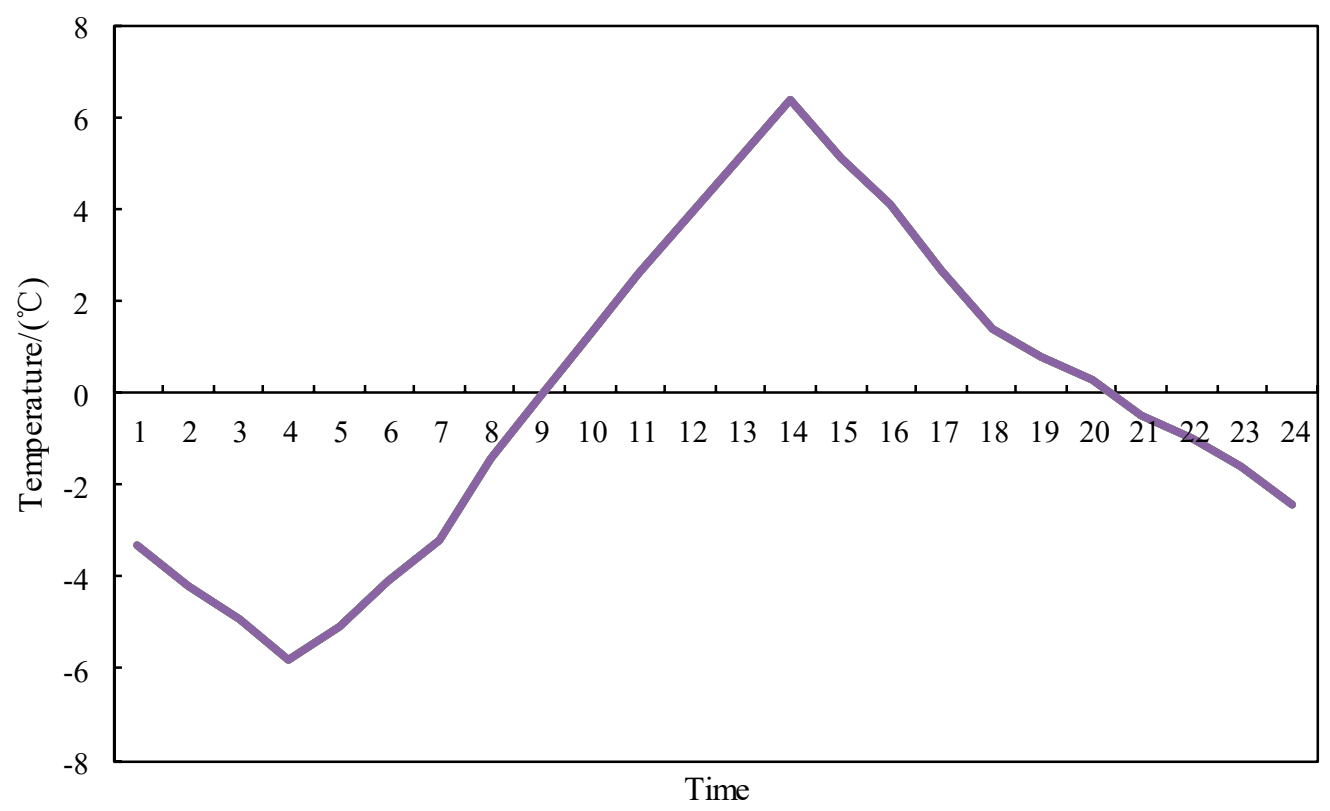

Figure A1. Outside temperature.

\section{References}

1. Gu, W.; Wu, Z.; Bo, R.; Liu, W.; Zhou, G.; Chen, W.; Wu, Z.J. Modeling, planning and optimal energy management of combined cooling, heating and power microgrid: A review. Int. J. Electr. Power Energy Syst. 2014, 54, 26-37. [CrossRef]

2. Wang, C.S.; Hong, B.W.; Guo, L.; Zhang, D.J.; Liu, W.J. A general modeling method for optimal dispatch of combined cooling, heating and power microgrid. Proc. CSEE 2013, 33, $26-33$.

3. Gu, W.; Wang, Z.H.; Wu, Z.; Luo, Z.; Tang, Y.Y.; Wang, J. An online optimal dispatch schedule for CCHP microgrids based on model predictive control. IEEE Trans. Smart Grid 2017, 8, 2332-2342. [CrossRef]

4. Huang, A.Q.; Crow, M.L.; Heydt, G.T.; Zheng, J.P.; Dale, S.J. The future renewable electric energy delivery and management (FREEDM) system: The energy internet. Proc. IEEE 2011, 99, 133-148. [CrossRef]

5. Barati, F.; Seifi, H.; Sepasian, M.S.; Nateghi, A.; Shafie-khah, M.; Catalão, J.S. Multi-period integrated framework of generation, transmission, and natural gas grid expansion planning for largescale systems. IEEE Trans. Power Syst. 2014, 30, 2527-2537. [CrossRef]

6. Ge, S.Y.; Liu, X.O.; Ge, L.K. Planning Method of Regional Integrated Energy System considering Demand Side Uncertainty. Int. J. Emerg. Electr. Power Syst. 2018, 20. [CrossRef]

7. Ameri, M.; Besharati, Z. Optimal design and operation of district heating and cooling networks with CCHP systems in a residential complex. Energy Build. 2016, 110, 135-148. [CrossRef]

8. Wang, J.J.; Zhang, C.F.; Jing, Y.Y. Multi-criteria analysis of combined cooling, heating and power systems in different climate zones in China. Appl. Energy 2010, 87, 1247-1259.

9. Ren, H.B.; Zhou, W.S.; Gao, W.J. Optimal option of distributed energy systems for building complexes in different climate zones in China. Appl. Energy 2012, 91, 156-165. [CrossRef]

10. Gu, W.; Lu, S.; Wang, J.; Yin, X.; Zhang, C.L.; Wang, Z.H. Modeling of the heating network for multi-district integrated energy system and its operation optimization. Proc. CSEE 2017, 37, 1305-1315.

11. Li, X.L.; Shan, F.Z.; Song, Y.M.; Zhou, H.M.; Liu, C.Q.; Tang, C.T. Optimal scheduling of multi-region integrated energy systems considering heat network constraints and carbon trading. Autom. Electr. Power Syst. 2019, 1-9. Available online: http://kns.cnki.net/kcms/detail/32.1180.TP.20190403.1720.048.html (accessed on 9 October 2019).

12. Wu, Q.H.; Zheng, J.H.; Jing, Z.X. Coordinated scheduling of energy resources for distributed DHCs in an integrated energy grid. CSEE J. Power Energy Syst. 2015, 1, 95-103. [CrossRef]

13. Morvaj, B.; Evins, R.; Carmeliet, J. Optimising urban energy systems: Simultaneous system sizing, operation and district heating network layout. Energy 2016, 116, 619-636. [CrossRef] 
14. Li, Z.G.; Wu, W.C.; Shahidehpour, M.; Wang, J.H.; Zhang, B.M. Combined heat and power dispatch considering pipeline energy storage of district heating network. IEEE Trans. Sustain. Energy 2016, 7, 12-22. [CrossRef]

15. Li, Z.G.; Wu, W.C.; Wang, J.H.; Zhang, B.M.; Zheng, T.Y. Transmission-constrained unit commitment considering combined electricity and district heating networks. IEEE Trans. Sustain. Energy 2016, 7, 480-492. [CrossRef]

16. Chai, B.; Chen, J.M.; Yang, Z.Y.; Zhang, Y. Demand response management with multiple utility companies: A two-level game approach. IEEE Trans. Smart Grid 2014, 5, 722-731. [CrossRef]

17. Apostolopoulos, P.A.; Tsiropoulou, E.E.; Papavassiliou, S. Demand response management in smart grid networks: A two-stage game-theoretic learning-based approach. Mob. Netw. Appl. 2018, 1-14. Available online: https://link.springer.com/article/10.1007/s11036-018-1124-x (accessed on 9 October 2019). [CrossRef]

18. Fu, Y.; Jiang, Y.L.; Li, Z.K.; Wei, C.F. Optimal economic dispatch for microgrid considering shiftable loads. Proc. CSEE 2014, 34, 2612-2620.

19. Zeng, M.; Wu, G.; Wang, H.J.; Li, R.; Zeng, B.; Sun, C.J. Resident Demand Side Response Control Strategy Considering User Satisfaction in the Background of Intelligent Power Consumption. Power Syst. Technol. 2016, 40, 2917-2923.

20. Wei, J.D.; Wang, J.X.; Gao, H.; Gao, X.H. Optimal Operation of Micro Integrated Energy Systems Considering Electrical and Heat Load Classification and Scheduling. In Proceedings of the IEEE Conference on Energy Internet and Energy System Integration (EI2), Beijing, China, 26-28 November 2017.

21. Zou, Y.Y.; Yang, L.; Feng, L.; Xu, Z.; Fu, X.H.; Ye, C.J. Coordinated heat and power dispatch of microgrid considering two-dimensional controllability of heat loads. Autom. Electr. Power Syst. 2017, 41, 13-19.

22. Jiang, Z.Q.; Hao, R.; Ai, Q.; Yu, Z.W.; Xiao, F. Extended multi-energy demand response scheme for industrial integrated energy system. IEEE Trans. Control Syst. Technol. 2018, 12, 3186-3192. [CrossRef]

23. Ali, M.; Koivisto, M.; Lehtonen, M. Optimizing the DR control of electric storage space heating using LP approach. Int. Rev. Model. Simul. 2013, 6, 853-860.

24. Geidl, M. Integrated Modeling and Optimization of Multi-Carrier Energy Systems; ETH: Zurich, Switzerland, 2007.

25. Gan, Z.H.; Zhu, X.J.; Wang, C.; Chen, Y. Ubiquitous energy internet-New energy Internet coupling with information and energy. Eng. Sci. 2015, 17, 98-104.

26. Chen, X.Y.; Xia, Q.; Kang, C.Q.; Teng, X.B. A Rural Heat Load Direct Control Model for Wind Power Integration in China. In Proceedings of the IEEE Power and Energy Society General Meeting, San Diego, CA, USA, 22-26 July 2012.

27. Liu, X.L.; Wang, Z.J.; Gao, F.; Wu, J.; Guam, X.H.; Zhou, D.M. Response behaviors of power generation and consumption in energy intensive enterprise under time-of-use price. Autom. Electr. Power Syst. 2014, 38, $41-49$.

28. Xu, X.C. Discussion on inspection and testing of ratio of electricity consumption to transfer heat quantity in central water heating system. Build. Sci. 2007, 23, 7-12.

29. Ren, H.B.; Gao, W.J. A MILP model for integrated plan and evaluation of distributed energy systems. Appl. Energy 2010, 87, 1001-1014. [CrossRef]

(C) 2019 by the authors. Licensee MDPI, Basel, Switzerland. This article is an open access article distributed under the terms and conditions of the Creative Commons Attribution (CC BY) license (http://creativecommons.org/licenses/by/4.0/). 